\title{
WestVirginiaUniversity
}

THE RESEARCH REPOSITORY @ WVU

Graduate Theses, Dissertations, and Problem Reports

2003

\section{Telecommunication of stabilizing signals in power systems}

Guillaume Julien Raux
West Virginia University

Follow this and additional works at: https://researchrepository.wvu.edu/etd

\section{Recommended Citation}

Raux, Guillaume Julien, "Telecommunication of stabilizing signals in power systems" (2003). Graduate Theses, Dissertations, and Problem Reports. 1306.

https://researchrepository.wvu.edu/etd/1306

This Thesis is protected by copyright and/or related rights. It has been brought to you by the The Research Repository @ WVU with permission from the rights-holder(s). You are free to use this Thesis in any way that is permitted by the copyright and related rights legislation that applies to your use. For other uses you must obtain permission from the rights-holder(s) directly, unless additional rights are indicated by a Creative Commons license in the record and/ or on the work itself. This Thesis has been accepted for inclusion in WVU Graduate Theses, Dissertations, and Problem Reports collection by an authorized administrator of The Research Repository @ WVU. For more information, please contact researchrepository@mail.wvu.edu. 


\title{
Telecommunication of Stabilizing Signals in Power Systems
}

\author{
by \\ Guillaume J. Raux \\ Thesis submitted to the \\ College of Engineering and Mineral Resources \\ at West Virginia University \\ in partial fulfillment of the requirements \\ for the degree of \\ Master of Science \\ in \\ Electrical Engineering
Professor Matthew C. Valenti, Ph.D., Chair
Professor Ali Feliachi, Ph.D., Co-Chair
Professor Mark Jerabek, Ph.D.

Lane Department of Computer Science and Electrical Engineering

\author{
Morgantown, West Virginia \\ 2003
}

Keywords: Deregulation, Networked control systems, Delays, OPNET Modeler, Delayed optimal controller, Network simulator, Delay jitter 


\title{
Telecommunication of Stabilizing Signals in Power Systems Guillaume J. Raux
}

\begin{abstract}
Deregulation of the power industry has occurred at a rapid pace, opening some promising competition between suppliers. The result of this competition should be to the benefit of customers.

Telecommunication plays a crucial role in integrating systems and ensuring smooth operation by way of exchanging data and information between various systems that are responsible for monitoring and control of the grid. For example, a Power System Stabilizer (PSS) controller could be placed at one of the generators and requires remote measurements. All the communication schemes within the network generate delays that are characterized in this project using OPNET Modeler. OPNET Modeler is also used to characterize the number of dropped packets.

As a case study, we consider a two-area four-generator (2A4G) and explore the role of communication delay on system stability. Those delays play an important role in the design of a controller that can help the damping of electro-mechanical oscillations between interconnected synchronous generators and therefore maintain the system stability. The network statistics are then imported into Simulink in order to visualize the speed of the shaft $w_{2}$ as a function of time. Additional communication options to the inter-area oscillation problem are offered at the end of the thesis (use of Virtual Private Network (VPN), use of fiber optic dedicated network, or the use of IPv6 protocol).
\end{abstract}




\section{Acknowledgments}

This work which highlights the importance of network delays when implementing a controller would not have been possible without the US DOE/EPSCoR WV State Implementation Award.

Foremost, I would like to thank Dr. Matthew C. Valenti who has been a terrific advisor and under whom I was a research assistant during my duration at the university. His insight, invaluable suggestions, and guidance helped shape this thesis. I would also like to thank Dr. Ali Feliachi, who led the team which was responsible for the DOE project, and Dr. Mark Jerabek, for their suggestions and help. I would also like to thank my colleagues and friends: Jian Sun, Bin Zhao, and Kirtan Modi at the wireless communications research laboratory (WCRL) and Amer Hasanović of the Advanced Power Engineering Research Center (APERC), for their help.

Finally, on a personal note, I would like to thank all my friends and my family for all their support and understanding throughout the process and to whom I dedicate this thesis. 


\section{Contents}

Acknowledgments $\quad$ iii

List of Figures vi vi vis

List of Tables vii

1 Introduction to Deregulated Power Systems 1

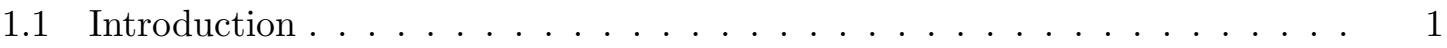

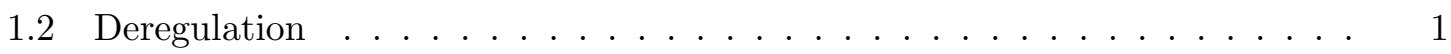

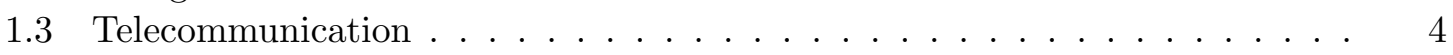

1.3 .1 Aspects: . . . . . . . . . . . . . . . . 4

1.3 .2 Issues: . . . . . . . . . . . . . . . . . . . 6

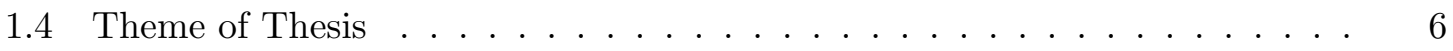

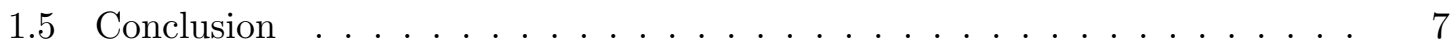

2 Modeling Communication Networks $\quad 8$

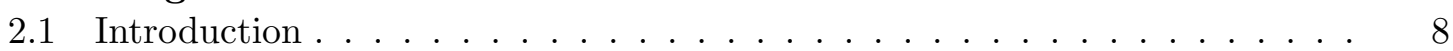

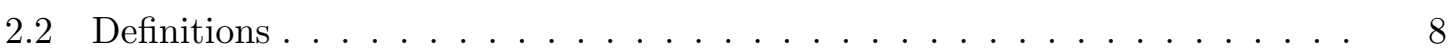

2.3 Protocols and Protocol Architectures . . . . . . . . . . . . . . . . . . 10

2.3 .1 Protocol Layering: . . . . . . . . . . . . . . . . . . . . . 11

2.3.2 Open Systems Interconnections: . . . . . . . . . . . . . . . . 12

2.3.3 Transmission Control Protocol/Internet Protocol: . . . . . . . . . . . 14

2.3.4 Discrete Event Simulators: . . . . . . . . . . . . . . . . 16

2.4 OPNET Modeler . . . . . . . . . . . . . . . . . . . . . 17

2.4 .1 What Is OPNET? . . . . . . . . . . . . . . . . . 17

2.4 .2 Why Use OPNET? . . . . . . . . . . . . . . . . . . . 17

2.4.3 OPNET Methodology: . . . . . . . . . . . . . . . . . 17

2.4 .4 A Case Study: . . . . . . . . . . . . . . . . . . . 2 20

2.5 Conclusion . . . . . . . . . . . . . . . . . . . . 24

3 The Two Area Four Generator System 25

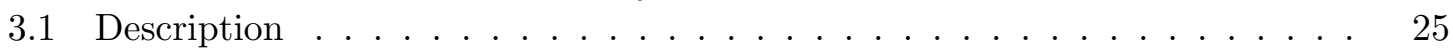

3.2 Why Use The 2A4G System? . . . . . . . . . . . . . . . . 26

3.3 Case Study with OPNET _. . . . . . . . . . . . . . . . 27

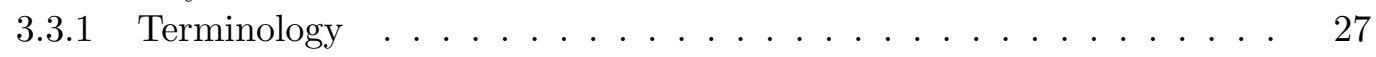


3.3.2 Modeling the Network: . . . . . . . . . . . . . . . . 29

3.4 Results........................... . 30

3.4.1 Packets Dropped: . . . . . . . . . . . . . . . . . 32

3.4 Delays: ...................... . . . . 32

3.5 Real-time closed loop system . . . . . . . . . . . . . . . . . . . 34

3.5.1 System Representation: . . . . . . . .......... . 34

3.5.2 Results: .......................... 35

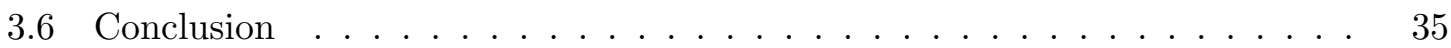

4 Other Solutions $\quad \mathbf{4 0}$

4.1 Dedicated Fiber Optic Network . . . . . . . . . . . . . . . . 40

4.1.1 Introduction: . . . . . . . . . . . . . . . . . 40

4.1 .2 Definitions: ..................... . . . 40

4.1.3 Signal Degradation and Delay: . . . . . . . . . . . . . . . 41

4.1 .4 Technical Data: . . . . . . . . . . . . . . . . . . . 42

4.1 .5 Conclusion: . . . . . . . . . . . . . . . . . . 43

4.2 Virtual Private Network . . . . . . . . . . . . . . . . . . . . 43

4.3 File Transfer Protocol . . . . . . . . . . . . . . . . . . . . 44

4.3.1 Introduction: . . . . . . . . . . . . . . . . . . 44

4.3 .2 IPv6: . . . . . . . . . . . . . . . . . . . . . 45

4.3.3 Conclusion:...................... 46

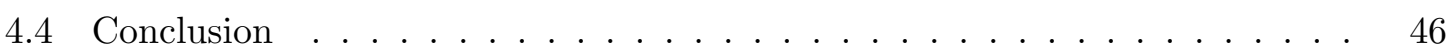

$\begin{array}{ll}\text { A List of Variables } & 47\end{array}$

B Acronyms $\quad 48$

$\begin{array}{lr}\text { References } & \mathbf{5 0}\end{array}$ 


\section{List of Figures}

1.1 The ISO system at work. . . . . . . . . . . . . . . . . . . . 3

1.2 The day-ahead and real-time energy market. . . . . . . . . . . . . . . 5

2.1 Basic representation of the Internet. . . . . . . . . . . . . . . . . . . . 9

2.2 OSI Architecture. . . . . . . . . . . . . . . . . . 13

2.3 Protocol architectures. . . . . . . . . . . . . . . . . 15

2.4 OPNET methodology. . . . . . . . . . . . . . . . . . . . . . . . . . . . . . . . . . . . . . . . .

2.5 OPNET network model. . . . . . . . . . . . . . . . . 19

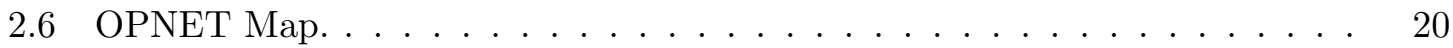

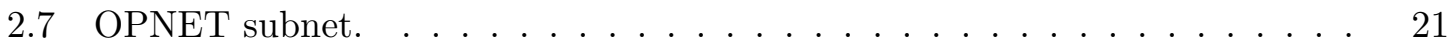

2.8 OPNET case study. ....................... . . . 22

2.9 OPNET object palette. . . . . . . . . . . . . . . . . 23

2.10 Traffic over the course of the day. . . . . . . . . . . . . . . . . . . . 24

3.1 The Two Area System. . . . . . . . . . . . . . . . . . 26

3.2 Detail of an Area. . . . . . . . . . . . . . . . . . . . . . 29

3.3 OPNET Model for the PSS Controller. . . . . . . . . . . . . . . 31

3.4 2A4G OPNET network. . . . . . . . . . . . . . . . . . . 32

3.5 Representation of the system with Simulink. . . . . . . . . . . . . . 37

3.6 Speed of the Shaft w2 as a function of the delays. . . . . . . . . . . . . . 38

3.7 Speed of the Shaft w2 as a function of the delays (when delay bigger than Dc). 39

4.1 A Virtual Private Netwok: A "pipe within the pipe"..... . . . . . . . 44 


\section{List of Tables}

3.1 Applications. . . . . . . . . . . . . . . . . . . . . 30

3.2 Number of Packets dropped for each type of connection. . . . . . . . . . . . 32

3.3 Delays by link. . . . . . . . . . . . . . . . . . . . . 33

3.4 Stability of the System. . . . . . . . . . . . . . . . 35

4.1 Power attenuation in $\mathrm{dB} / \mathrm{km} \ldots \ldots \ldots \ldots$. . . . . . . . . . . . 41

4.2 Data rates for different fiber optical cables. . . . . . . . . . . . . . . 43 


\section{Chapter 1}

\section{Introduction to Deregulated Power Systems}

\subsection{Introduction}

Electricity is an indispensable commodity. It runs our computers, lights our lamps and heats our French roast coffee, but mainly allows the industry to run. Whenever we flip a switch, the electricity we need is instantly supplied. We count on that reliability. One of the compelling topics currently faced by the electric power industry is the prospect of substantial change in its structure and organization [1] resulting from more competition that is supposed to benefit the customers. These coming changes can be easily compared to the dramatic changes which have taken place in the telecommunications and natural gas industries [2] years ago. The electric power industry, the last major regulated energy industry in the United States, is changing to be more competitive [3].

In some states, retail electricity customers already have the opportunity to choose their electricity provider. New electricity trading markets, which were previously nonexistent, are now operating in all regions of the country.

\subsection{Deregulation}

The nature of electricity presents unique challenges because it cannot be stored in large quantity, so it must be produced the moment it is needed. The requirements for 
reliability mean that enough reserves must be available at any time. However, the exact amount of electric power needed is almost impossible to predict [4].

Deregulation in the power industry is occurring at a rapid pace. Deregulation promises to stimulate competition between suppliers, to the benefit of the customers, improving the power quality and reliability [5]. The number of independent producers and power marketers competing in these new retail and wholesale power markets has increased substantially over the past few years. Different structures have been created in order to assure a competitive oligopolistic market that would ensure sufficient electricity generation, distribution and monitoring of power at a competitive price [6]:

- ISO Organization: The Independent System Operator (ISO) (Fig. 1.1) is mainly responsible for ensuring the stability and availability of power within the grid. It manages the traffic and provides feedbacks to the participants in the market [6].

- RTO Organization: The Regional Transmission Owner (RTO) structure is responsible for investment in electric transmission and generation infrastructure.

- Power Exchange: The Power Exchange (PX) is responsible for the creation of a competitive spot energy market. The PX structure takes care of scheduling, settlement, trading platforms and brokerages, market infrastructure systems, and consulting [7].

In most cases, the PX acts as a Scheduling Coordinator (SC). The ISO work is shown in Fig. 1.1. At a control center of an ISO, an expert staff monitors the grid constantly adjusting the production to accommodate the changes in demand. The staff works on "what if" scenarios to be prepared for any event (equipment failure, extreme weather conditions, and emergency situations). The staff is also in charge of scheduling import and export transactions. There are two different kinds of markets available to electric suppliers that work simultaneously: the day-ahead and the real-time energy market (Fig. 1.2). The two energy markets are discussed below for the California ISO case.

- The day-ahead The ISO receives bids for the sale and purchase of energy for the next day between 8:00 am and noon [7]. At noon, the window for entering day-ahead 

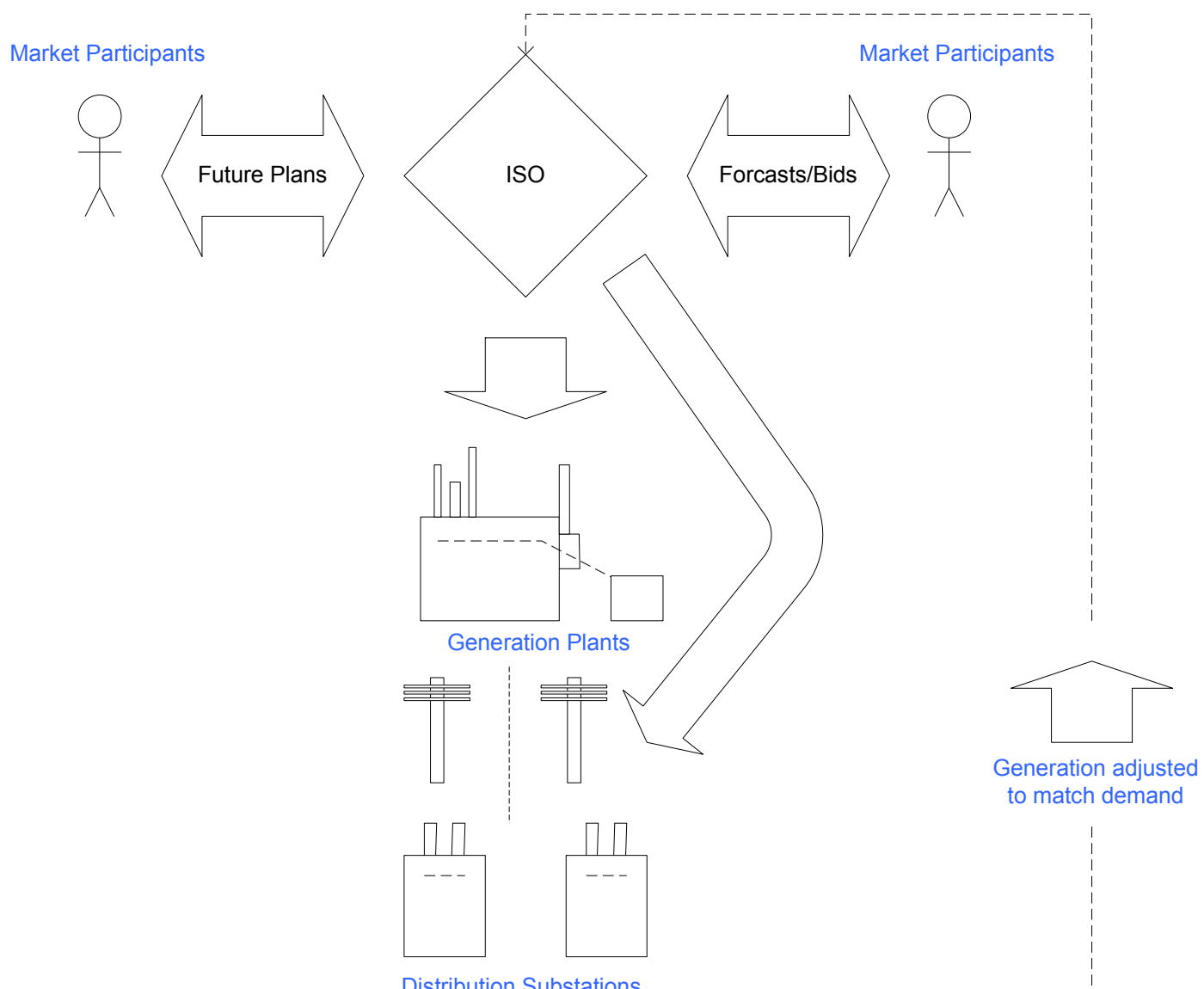

ion Substations
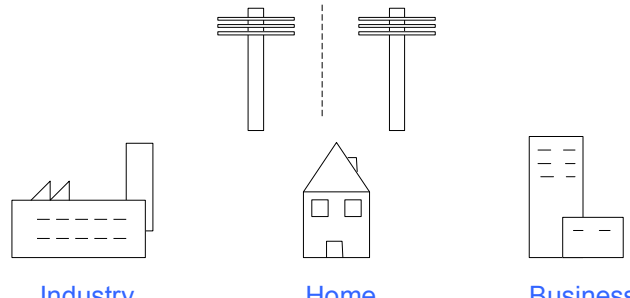

Industry

Home

Business

Figure 1.1: The ISO system at work. 
closes. Between noon and 2:00 pm, the ISO reviews the latest demand forecast, receives transaction data for imports and exports, and performs initial evaluation of bids for the next day. Then, from 2:00 pm to 4:00 pm, the ISO performs the final evaluation of the bids and offers. It issues schedules for the next day by 4:00 pm. All the schedules indicate the output levels required at each generating plant for each hour. It also indicates the marginal price that will be paid. The 4:00 pm to 6:00 pm slot period is a rebidding period. All the generators that have not been selected previously can make a revised offer to supply the next day. The last time slot, from 6:00 pm to 8:00 pm, the ISO evaluates bids and offers to optimize the operating plan (see Fig. 1.2).

- Real time energy market Throughout the day, the ISO continues to adjust the electric generation output to match the demand. The real time market is settled based on deviations between actual demand and the bid supply.

\subsection{Telecommunication}

Telecommunication plays a crucial role in integrating systems and ensuring smooth operation by way of exchange of data and information between various systems that are responsible for monitoring and control of the grid.

\subsubsection{Aspects:}

- ISO: An ISO structure is a centrally dispatched and coordinated system. One of the main necessities of the system is transportation of information from remote areas to the ISO structure. In order for an ISO to ensure neutrality and independence, the ISO needs information from bidders (market participants) and from generating plants. The ISO also needs feedback from industries, home, and businesses in order to adjust the production to match the demand. All the communication is represented by arrows in Fig. 1.1. Since the ISO is in charge of determining the future expansion of the electric system, it needs to be able to interact with the board of managers allowing them to decide on a potential expansion of the grid. 


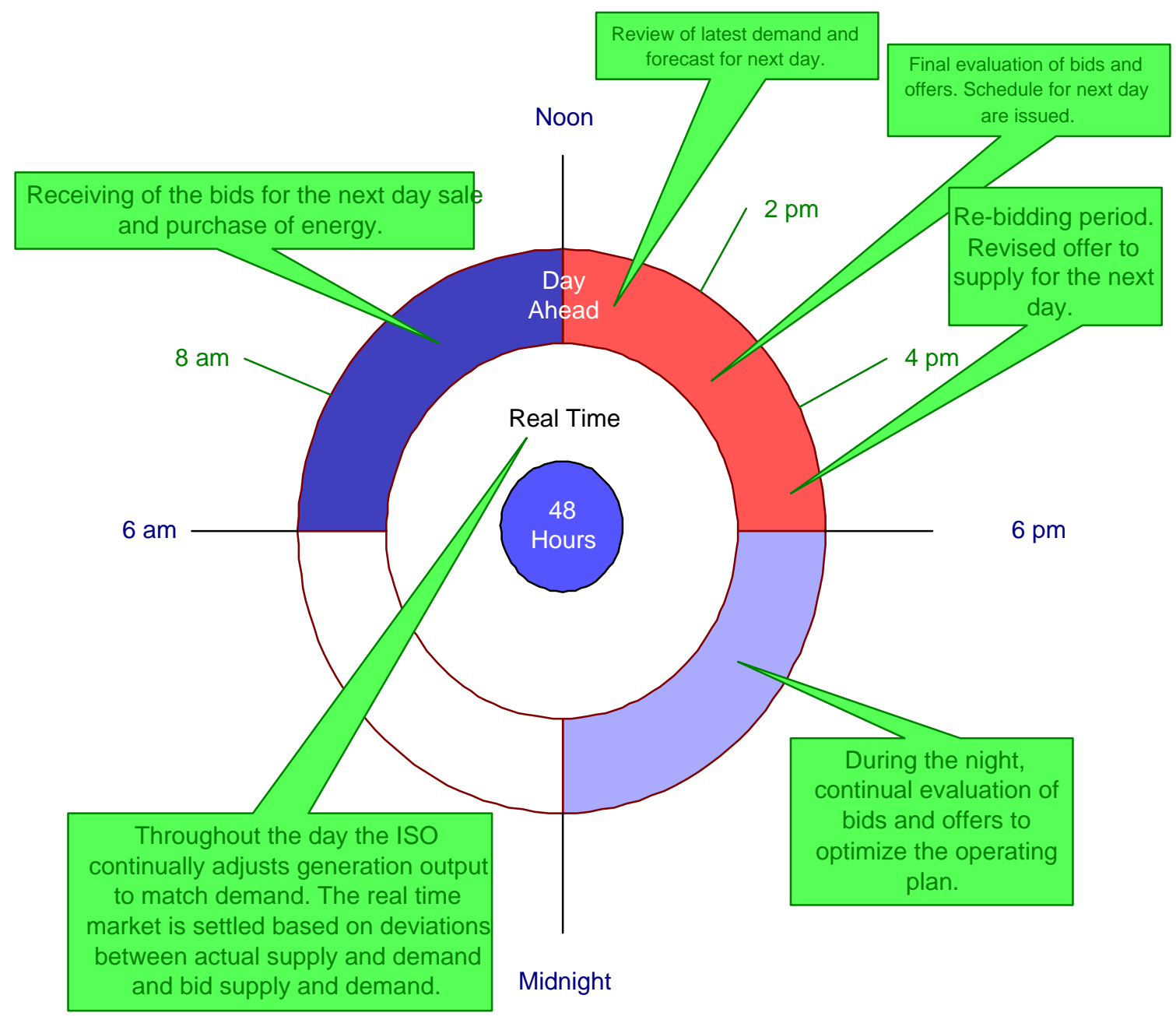

Figure 1.2: The day-ahead and real-time energy market. 
- Control: Inter-area oscillation is a major problem that can be solved by using appropriate control structure either at the transmission line or at the power plants. The controller might have to use remote measurements to minimize the inter-area oscillations. The measurements need to be transported from a remote area to the controller with minimum delay.

\subsubsection{Issues:}

The scale of the system makes the transport of information a serious issue. Like every large system, delays generated by the links are the most prevalent problems. Controllers, ISO structure, power plants, all need to be "fed" with information. Because information is not necessarily local, a communication link needs to be implemented. The communication needs to be reliable and fast in order for the system to be able to work more efficiently. A lot of different links (wired, or wireless) can be used to carry the information.

\subsection{Theme of Thesis}

The purpose of this thesis is to study the delays generated by different communication links and to use the results obtained for the design of a controller to dampen inter-area oscillations. One of the tools which we have used to collect the delays is the OPNET Modeler software.

Because communication delays are playing an important role, a special consideration is given to their calculation. Transmission of crucial data can have a negative impact on control procedures because it might be too late for an action to be initiated to control power grid instabilities. Various communication links can be utilized for the transport of data including 56K modem line, DS-1, DS-3, 100BaseT, and OC-12 fiber optic cable. Each communication link has its own advantages and drawbacks associated with it. This thesis looks at various communication links and the delays associated with each type of link.

This thesis also looks at the effect that those delays have on the Power System Stabilizer (PSS) controller. This thesis intends to define the critical delay time $\left(D_{c}\right)$ that needs to be respected when implementing a control scheme within the Two Area Four Generator system $(2 \mathrm{~A} 4 \mathrm{G})$. The end of the thesis is dedicated to the search for the best 
overall link and the exploration of an alternative solution for the transfer of files. The notion of optimal is subjective and is defined within the thesis as the best ratio delay/cost.

\subsection{Conclusion}

This chapter discussed the need for deregulation in the power industry and its consequences. This chapter also highlights the need for an efficient communication link. Finally the theme of the thesis was presented. 


\section{Chapter 2}

\section{Modeling Communication Networks}

\section{$2.1 \quad$ Introduction}

In 1969, the Advanced Research Projects Agency (ARPA) a branch of the US Department of Defense, established the ARPANET (Advanced Research Projects Agency Network) for the interconnection of computers and terminals. ARPANET was the first packet-switched network [8], and later became the Internet (Fig. 2.1).

\subsection{Definitions}

Data communication occurs between two devices that are directly connected by some form of point-to-point transmission medium [9]. The two major categories into which communications networks are conventionally classified are wide-area networks (WANs), and local-area networks (LANs). LANs allow computer systems in a building or within an enterprise to be connected together and share common files, sources of information, and/or applications. Usually LANs are administrated by some type of organization that owns the devices attached to the network. WANs, on the other hand, have been traditionally considered to be those that cover a large geographical area. WANs consist of a number of interconnected switching nodes. A transmission from any device is routed through these internal nodes to the specified destination device. These nodes provide a switching facility to move packets of data from node to node until they reach their destination. WANs have 


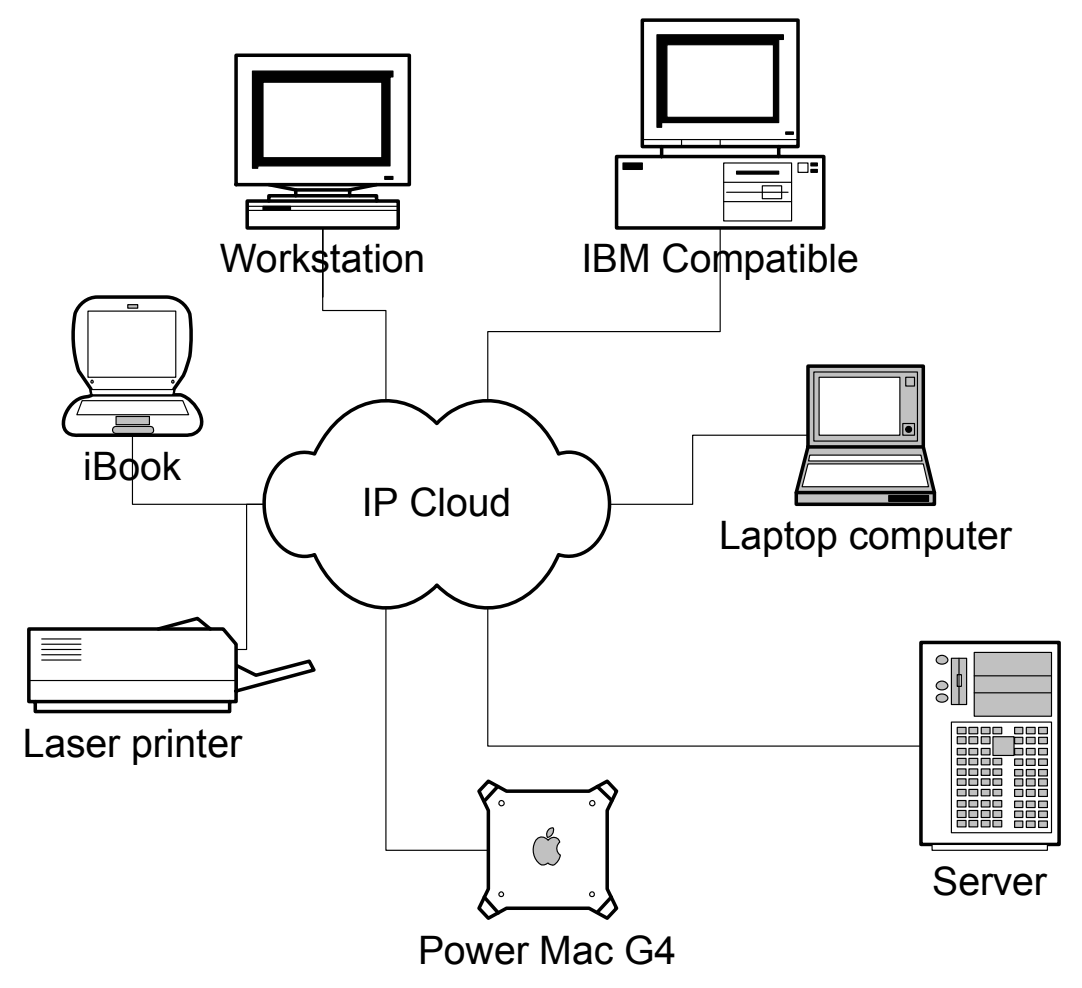

Figure 2.1: Basic representation of the Internet.

been implemented with two different technologies: packet switching and circuit switching. Today, two other technologies, asynchronous transfer mode (ATM) and frame relay, are assuming those roles [10]. All these different technologies are explained in more detail below.

- Circuit Switching: In this kind of switching, a dedicated communication path is established between two stations through the nodes of the network (end-to-end communication between two hosts). Data generated at the source are transmitted along the dedicated path as fast as possible. At each node, incoming data are routed to the appropriate channel without delay. This technology is mainly used in telephone networks. 
- Packet Switching: In this case, there is no dedicated transmission capacity along the path, rather data are sent out in a sequence of small chunks, called packets. A packet is a small piece of the source message. Each packet is transmitted through the entire network, via routers, from node to node at a rate equal to the full transmission rate of the link. At each node, the entire packet is received, stored briefly, and then transmitted to the next node.

- Frame Relay: The frame relay approach was developed in the early 1980's and deployed in the 1990's throughout North America as a public packet-switching technology. Frame relay is a fiber-based system that has very low bit error rates (BER) and high data rates. Frame relay networks have been designed to operate efficiently at data rates up to $200 \mathrm{Mbps}$ [9]. Frame relay uses variable-length packets, and achieve high data rates by stripping most of the overhead involved with error control (additional data bits added at the end of each packet).

- ATM: Asynchronous transfer mode, sometimes referred to as cell relay, is a result of all the developments in circuit switching and packet switching over the past few decades. ATM is an evolution of frame relay, but ATM technology uses fixed-length packets called "cells". ATM technology does not provide too much control overhead and can easily achieve rates up to 100's of Mbps [8].

\subsection{Protocols and Protocol Architectures}

During the process of exchanging data between computers, terminals, and/or other data processing devices, a "level" of comprehension is required in order for the data to take the right path. The process of logically dividing the communication process into distinct steps is called a protocol. A protocol is used for communication between entities in different systems. There are three key elements in a protocol [9]:

- Syntax: Includes data format and signal levels.

- Semantics: Includes control information for coordination and error handling.

- Timing: Includes speed matching and sequencing.

In order to exchange data between computers a high degree of cooperation is needed. The logic is implemented not as a single module but instead the task is broken 
down into subtasks, each of which is implemented separately that are referred to as "protocol architecture". The main two architectures are Transmission Control Protocol/Internet Protocol (TCP/IP) and Open System Interconnections (OSI). Those architectures are composed of 5 and 7 layers respectively, and will be described later in more detail in the chapter.

In very general terms, communications can be said to involve three types of agents applications (e.g. file transfer), computers, and networks. Computers are connected to networks, and the network transfers all the data from one computer to another one. This process involves getting the data to the computer in which the application resides and then getting it to the intended application within the computer.

\subsubsection{Protocol Layering:}

Protocol layering is a common technique to simplify networking designs by dividing them into functional layers, and assigning protocols to perform each layer's task. The original message is passed along the protocol stack, and the protocols at one layer rely on and use the services of the layer below.

In order for one layer to inter-operate with the layer below it, the interfaces between the two layers must be precisely defined. Standard bodies define the interfaces between adjacent layers and allow the person that develops networking hardware and/or software to implement the interior of a layer as they want. Therefore, if one comes up with an improved implementation layer, the old layer could be replaced without stopping the inter-operation between those layers.

In a computer network, each layer can perform a generic set of tasks [10]:

- Error Control: Any technique that will detect or correct errors making the network communication between two elements more reliable.

- Flow Control: This mechanism avoids overwhelming a slower peer.

- Segmentation and Reassembly: Segmentation is the process of breaking large 
message into small packets. The receiving side is the side that reassembles the packets into the original large message.

- Media Access Control: The MAC protocol allows multiple users to share the same media/channel.

- Connection Setup: It provides handshaking with a peer.

Protocol layering has both advantages and drawbacks. The main advantage is that protocol layering makes it easier to design complex systems, and facilitates the re-use and design of software/hardware. On the other hand, one of the main drawbacks of layering is that one of the layers may duplicate lower-layer functionality. For example, many protocol stacks provide error control recovery on both a link basis and an end-to-end basis. Another significant drawback is that functionality at one layer could need information from another layer, violating the purpose of separation of layers.

\subsubsection{Open Systems Interconnections:}

The OSI protocol [11] shown in Fig. 2.2 was developed in the late 1970s by the International Standards Organization (ISO) with the association of the International Telecommunications Union (ITU). The OSI model consists of seven layers. Layers 1 to 3 are part of the network connection, while layers 4 to 7 are part of the data originator (source) or recipient (destination) [8]. This kind of configuration allows modifying one layer at a time without affecting the other layers.

The layers are described as followed:

1. Physical: The physical layer is where the bit is inserted into the network. The physical layer can be seen as a noisy "bit pipe". The physical layer includes electrical and mechanical standards (e.g. 1.430 BISDN, ISO-8877 ISDN Connector, Ethernet 802.3).

2. Data Link: The data link layer structures the data into frames. Frames include rules controlling information for synchronization, error detection/correction, and the 


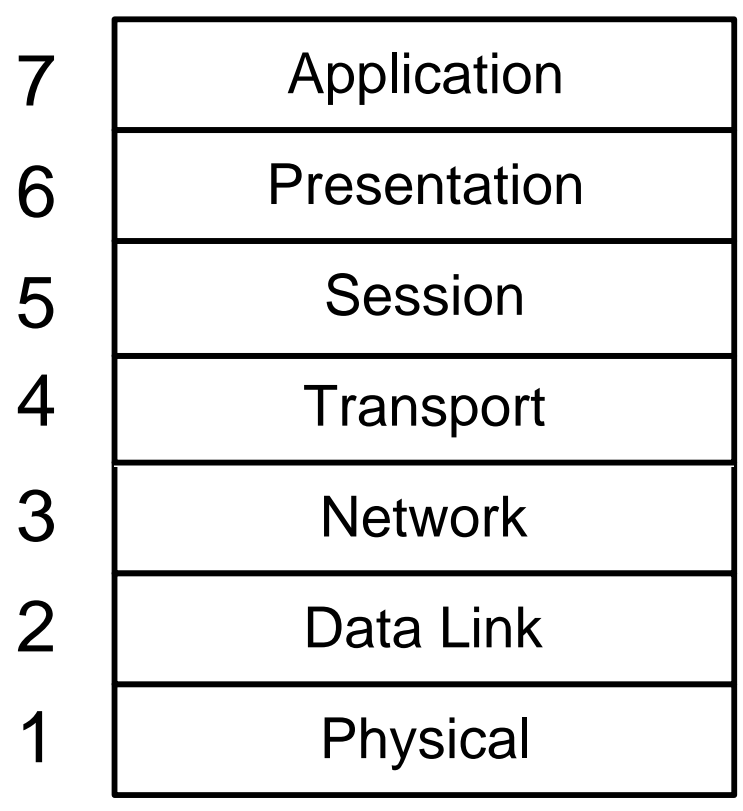

Figure 2.2: OSI Architecture.

source's and destination's address. The data link layer is also used to send and receive acknowledgements in order to confirm that the frame was received correctly on a peer-to-peer basis. The data link layer also makes available the connection between a "slow" destination and a "fast" source. It also makes sure that proper data rate is used, and the destination is not overwhelmed by the data flow.

3. Network: The network layer takes care of the routing of data from node to node, and the logical addressing. The network layer is a MAC independent interface.

4. Transport: The transport layer either breaks the message data from the session layer into packets or takes the packets from the network layer and connects them to form a message. The transport layer provides reliable data transfer and manages the flow of data in case of network congestion. The transport layer is also involved in receiving and in sending acknowledgments.

5. Session: The session layer establishes connections (or re-established a failed connec- 
tion) between computers. It also takes care of video and audio within an application.

6. Presentation: The presentation layer performs data formatting (e.g. code conversion, encryption/decryption, and compression/decompression).

7. Application: The application layer is used to interface the computer to the OSI system. It includes file transfer programs, email, and web browsers.

In practice, this seven layers can be integrated at various degrees into a single piece of hardware.

\subsubsection{Transmission Control Protocol/Internet Protocol:}

The TCP/IP protocol architecture is the most widely used inter-operable architecture. It was developed in conjunction with the original ARPANET and preceded the ISO model. The TCP/IP is not a standard protocol like the ISO one, because it consists of a large collection of protocols that have been issued as Internet standards by the Internet Activities Board (IAB) [9].

Despite its OSI-like structure the TCP/IP protocol has no "official" model. It can be organized into five relatively independent layers (see Fig. 2.3).

- Physical Layer: The physical layer covers the physical interface between the data transmission device (e.g. workstations, computers) and the transmission medium or network. This layer is concerned with specifying and the characteristics of the transmission medium, the data rate, and the nature of the signal.

- Network Access Layer: The network access layer is concerned with the exchange of data between the computers and the attached network. The sending computer must provide the destination's address so that the network can route the data in an efficient manner. 


\begin{tabular}{|c|c|}
\hline Application & \\
\hline Presentation & Application \\
\hline Session & \\
\hline Transport & $\begin{array}{c}\text { Transport } \\
\text { (host-to-host) }\end{array}$ \\
\hline Network & Internet \\
\hline & \multirow{2}{*}{$\begin{array}{c}\text { Network } \\
\text { Access }\end{array}$} \\
\hline Data Link & \\
\hline Physical & Physical \\
\hline
\end{tabular}

OSI TCP/IP

Figure 2.3: Protocol architectures.

- Internet: The Internet layer provides routing across multiple networks. This protocol is implemented at every router used by the message during its transport across the communication link.

- TCP: The TCP protocol is also called the host-to-host or transport layer. This mechanism provides reliability to the system (it is independent of the nature of the application).

- Application Layer: The application layer contains all the logic needed to support the various user applications. For each different application a particular module is used. 
In chapter 4, the difference between IPv4 and IPv6 will be discussed in detail.

\subsubsection{Discrete Event Simulators:}

A discrete event simulator is a piece of software that has the ability to simulate continuous events as a sequence of discrete events. Numerous discrete event simulators (free and commercial) are now available on the market. They are designed to be general enough to serve a wide range of discrete event simulation tasks, like a computer simulation of a network [12]. Most of these discrete event simulators are easy to use because of a well-defined interface. All of them include a simulation kernel library and a compiler. Some popular free network simulators are as follows [13]:

- NS2: NS2 is a discrete event simulator targeted at networking research. NS2 provides substantial support for simulation of TCP, routing, and multicast protocols over wired and wireless (local and satellite) networks. NS2 is free, runs on Linux/Unix and it was made possible due to the collaboration of LBL, Xerox PARC, UCB, and USC/ISI.

- Ptolemy: This discrete event simulator (java based) was developed at the University of California at Berkeley. It operates mainly under Linux and/or UNIX. The Ptolemy software helps the modeling, simulation, and design of concurrent, real-time, embedded systems.

- Omnet++: Omnet ++ is also a free discrete event simulator software development environment written in $\mathrm{C} / \mathrm{C}++$. It was mainly developed by A. Varga from MIT. Within the software there is a useful list of modules and workshops. It works under all Linux, UNIX, and NT.

- Cnet: Cnet network simulator allows experimentation with the data-link layer, network layer, routing, and transport layer networking protocols. This network simulator works under both Linux and UNIX. 


\subsection{OPNET Modeler}

\subsubsection{What Is OPNET?}

OPNET Modeler is a discrete event simulator that allows one to simulate computer and data networks. OPNET Modeler is mainly an environment for building protocols and device models [14]. The OPNET Modeler environment is also used to plan changes by illustrating how the networked environment will perform, and is also an environment that includes hundreds of pre-built models used to study performance changes of the network.

\subsubsection{Why Use OPNET?}

The use of OPNET software (based on $\mathrm{C} / \mathrm{C}++$ functions) is an easy solution for network management because of its close tie to companies, service providers, and network equipment manufacturers. OPNET Modeler provides a suite of vendor module libraries. It also has the flexibility for the user to create new models or derive models from an existing database.

\subsubsection{OPNET Methodology:}

The methodology for a general simulation is first to understand the proposed system in order to model it accurately (flow chart, diagram, etc). The next step is to define what the needed throughputs for the simulation are. By choosing the aspects that need to be modeled, one will be able to determine better objectives and define the level of detail or "granularity" required in its model.

OPNET Modeler, through its flexibility, allows some portion of the model to have fine granularity, while other portions have coarse granularity. The following step in the simulation is to define the data inputs and outputs. In OPNET Modeler, the input refers to a predefined aspect of the model (such as connectivity) or may be more parametric (such as traffic generation rate).

To study the effects on a system when using a discrete event simulator, one needs to keep most inputs constant and vary one or two over a range. OPNET Modeler allows one to present the output under different aspects: graphs, animations, tables, numerical 


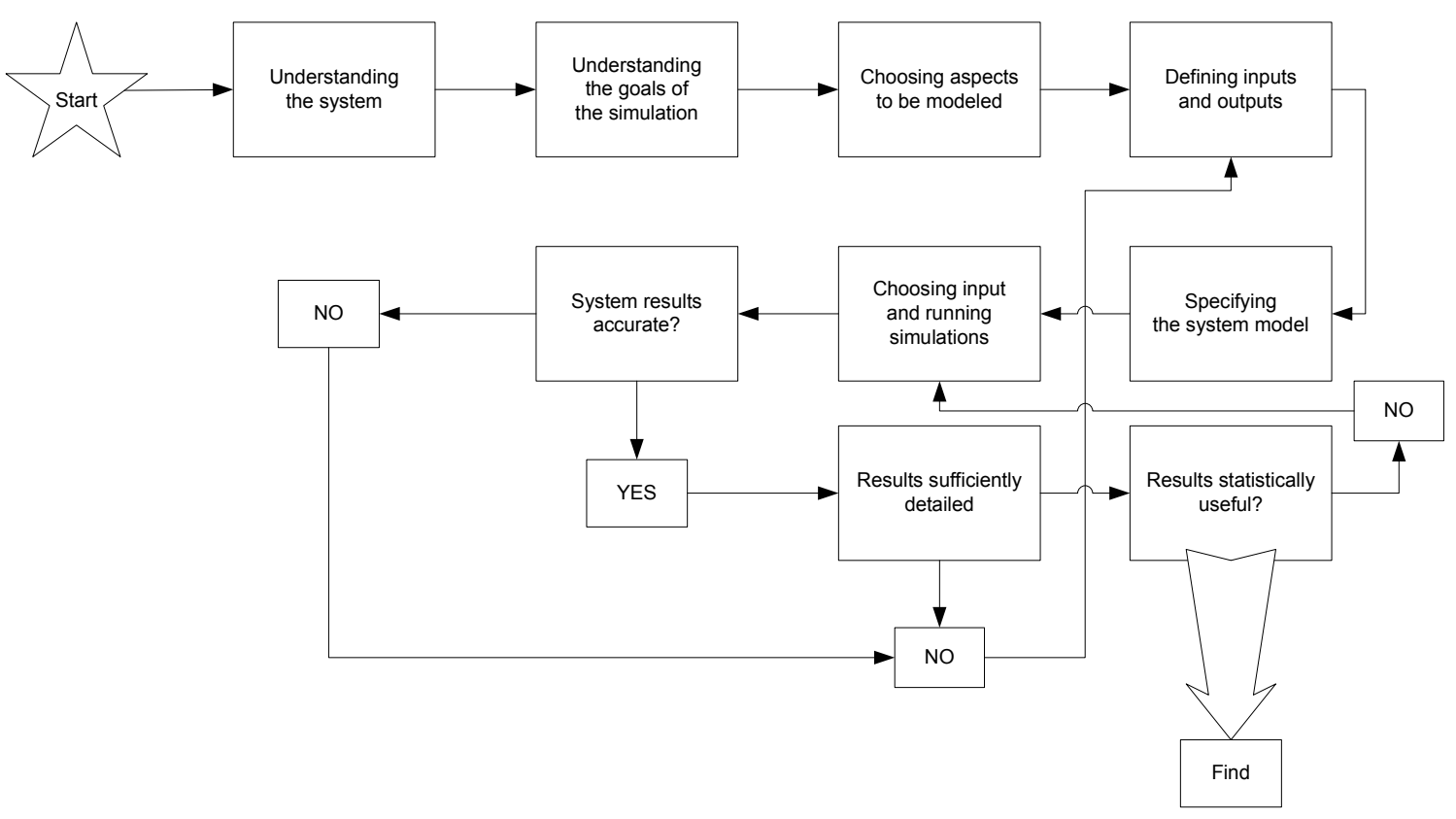

Figure 2.4: OPNET methodology.

values and/or web reports. The output results should be checked for accuracy, robustness and, show enough details. A good summary of the methodology is the chart shown in Fig. 2.4 .

OPNET Modeler uses a project-and-scenario approach to modeling networks. A project is a collection of related network scenarios in which each explores a different aspect of network design. All projects contain at least one scenario. A scenario is a single instance of a network. Typically, a scenario presents a unique configuration for the network, where configuration can refer to aspects such as topology, protocols, applications, baseline traffic, and simulation settings.

The project/scenario workflow is as follows: first create a project, then create a baseline scenario, and finally duplicate the scenario. When creating a baseline scenario, one needs to import or create a topology, import or create traffic, choose results and report to be collected, run the simulation, and view the results. The duplicate of the scenario allows one to make changes, re-run the simulation, and compare the results. 


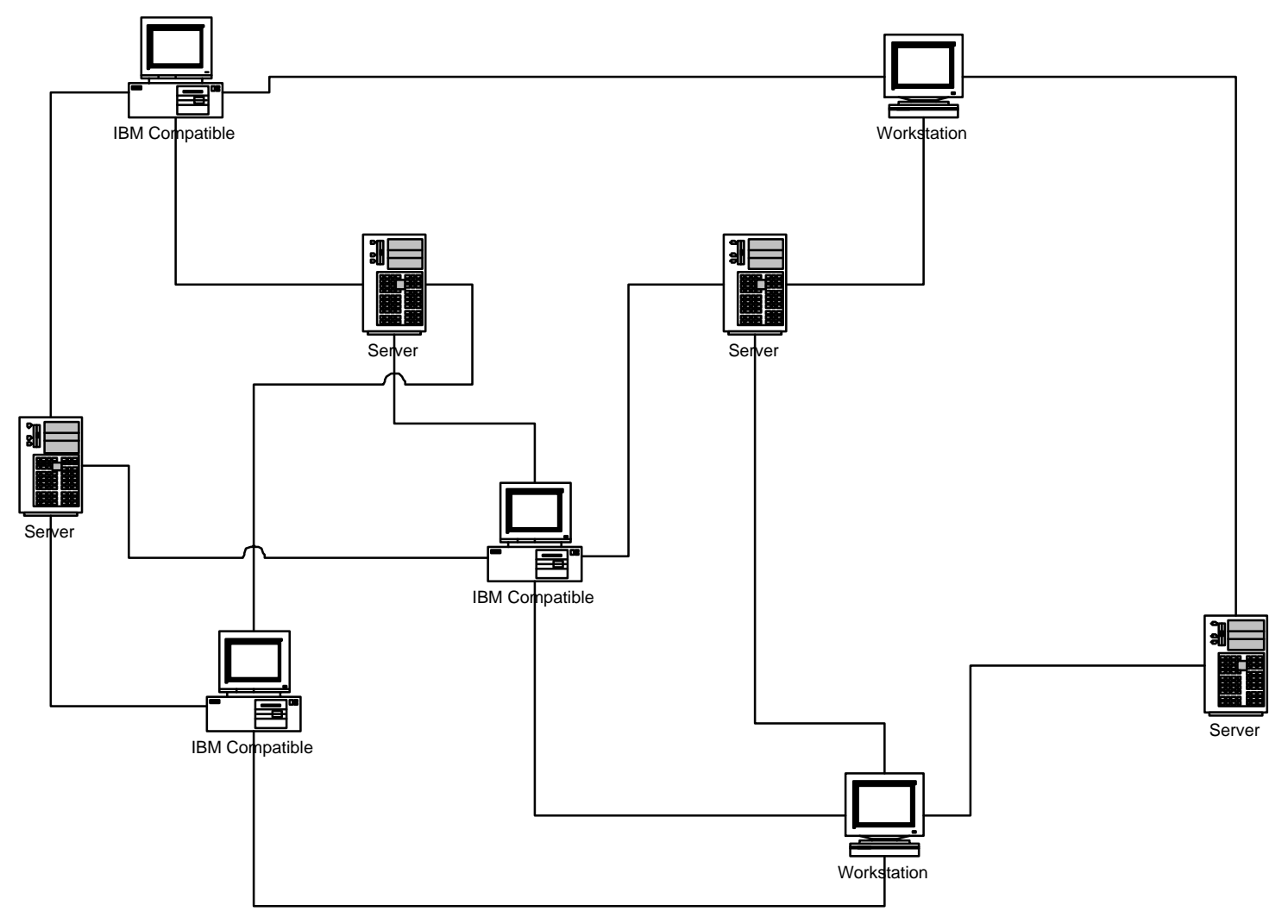

Figure 2.5: OPNET network model.

The networks are modeled via the graphical project editor. Several tools are included in OPNET Modeler to intuitively map from network specifications to a modeled network Fig. 2.5. OPNET Modeler includes several maps Fig. 2.6 that can be loaded as backgrounds for network models.

Map backgrounds, Fig. 2.6, provide a physical context for model specification. Models are more easily interpreted when set in a proper geographical context. The distance between nodes can be a factor in affecting simulation results. OPNET Modeler uses subnets, Fig. 2.7, within the network as a powerful mechanism for breaking down system's complexity through abstraction. Subnets can be used to segment networks into distinct parts based on proximity, connectivity, or architecture. Figure 2.7 represents few elements (on the right of the figure) contained within a subnet (on the left of the figure). 


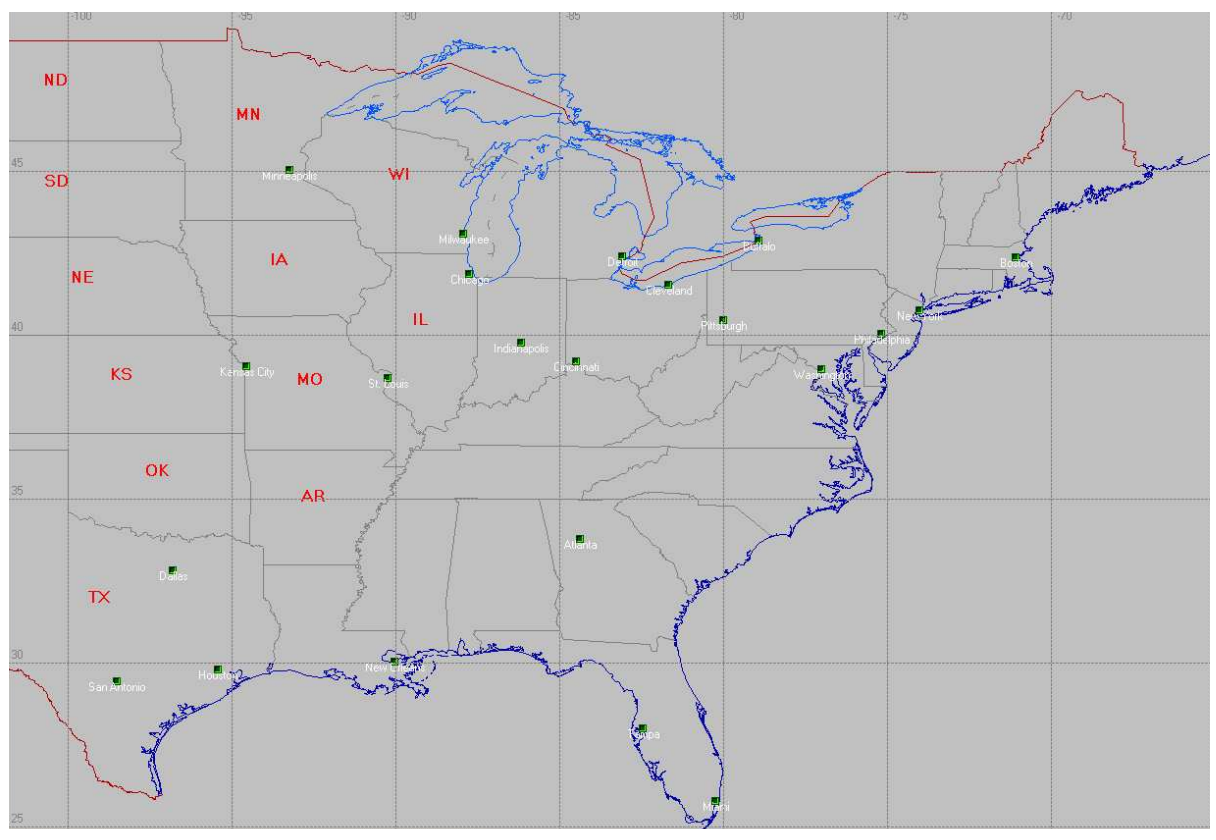

Figure 2.6: OPNET Map.

A subnetwork abstracts network components specified within it into a single object. A subnetwork represents identical constructs in an actual network. It has no behavioral aspects, but simplifies representation of large networks. The models formed with OPNET Modeler are created using published protocol standards and other used vendor implementations (IEEE specifications -e.g. 802.11a-, ATM Forum Specification -e.g. UNI 3.1- ).

The "model library" contains a variety of objects used to create networks like: traffic generator (workstations, servers, stations), network devices (hubs, bridges, switches, routers), links (SONET, PPP, FDDI, 10baseT, DSL), vendor device models (Cisco systems, 3Com, Nortel, Lucent, HP).

\subsubsection{A Case Study:}

OPNET can be used to model the network reaction when using background utilization. The following network case study helps to visualize those results:

- Link background utilization: Background utilization is used to model existing traffic on a link instead of explicitly modeling each packet. It is possible to change the background traffic over the course of the simulation. 

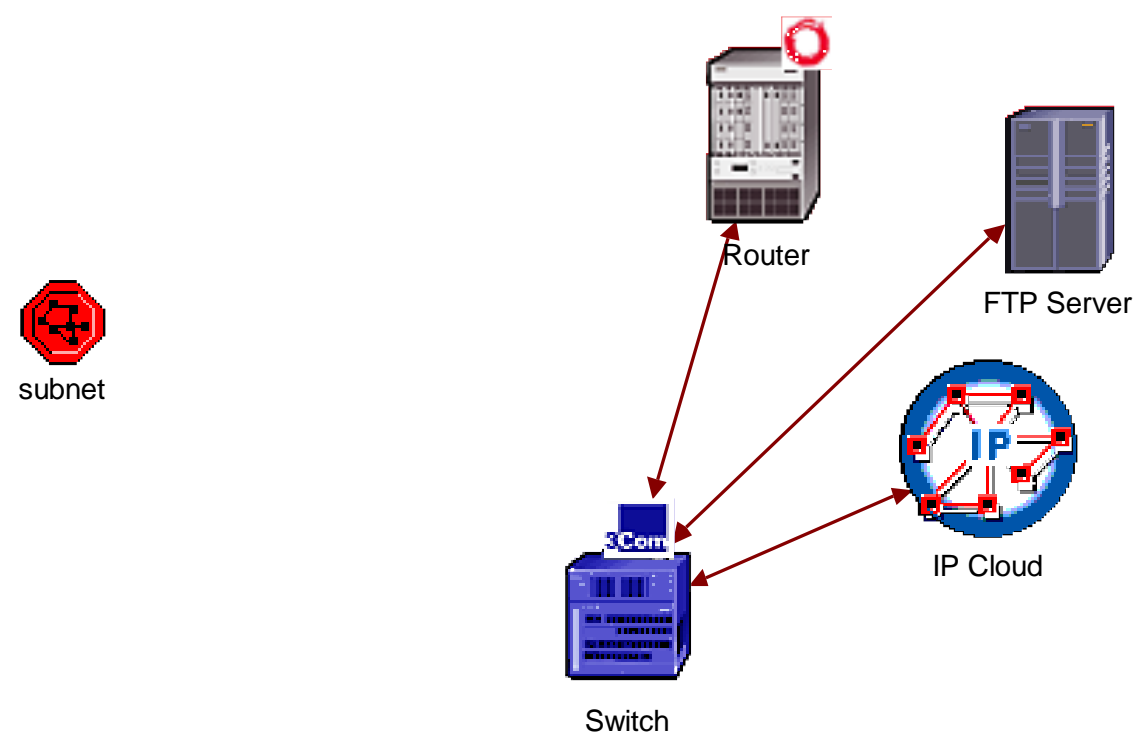

Figure 2.7: OPNET subnet.

In the following case study, the goal is to model an East Coast Company's WAN. The company has offices in Philadelphia, Atlanta, Washington D.C., and Boston. The center of the network will be located in Washington D.C. (see Fig. 2.8). The company is trying to visualize the effect of background traffic on the performance of FTP. To do this, we need to first model the performance of the network without any background traffic and then with background traffic.

The background traffic will consist of the use of database, email, file transfer, file print, Telnet session, video conference, voice over IP (VoIP), and web browsing. For all those applications, the user at the workstation will have the option of both heavy and light use. For the first 2 hours (from 8:00 am to 10:00 am), the background utilization will be setup to $40 \%$. Then for the next 3 hours, it will be increased to $90 \%$, and finally it will be decreased again for the next 6 hours to $70 \%$.

Now that the scenarios are configured, we will need to collect statistics (data) in order to be able to make any type of comparison. The most relevant statistics for this network are the utilization statistics for the links and the global FTP download time for the network. Also, it could be interesting to know if at any switch the network will not be 


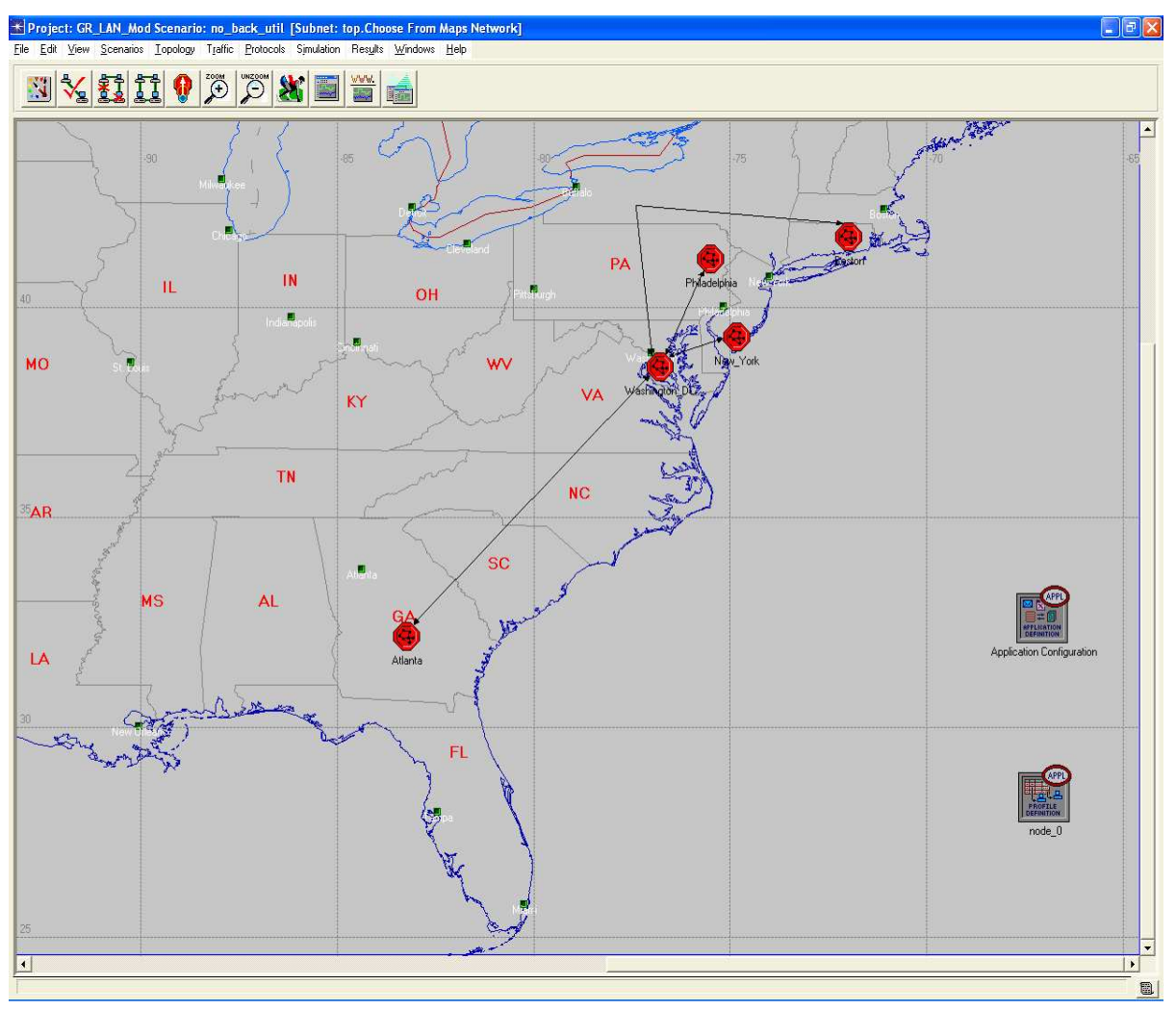

Figure 2.8: OPNET case study.

able to handle all the packets. Therefore, one should also look at the number of dropped packets within the network.

- Setting up the scenario: This first step helps to specify the overall context for the network. Within this step, one will have to specify if one plans on using a map in the background for the network, and what kind of "tools" one is going to use.

All those "tools" are already pre-defined within a library, and they are available in the "Object Palette" Fig. 2.9. The "Object Palette" allows you to specify what standard (model list, e.g. LAN and/or Internet...) and what object you are going to use. For the example we will need to use different links (e.g. fiber optic links, coaxial cable,...), servers, and routers. OPNET offers the option of configuring your object palette list by link models, node models, path models, and demand models. 


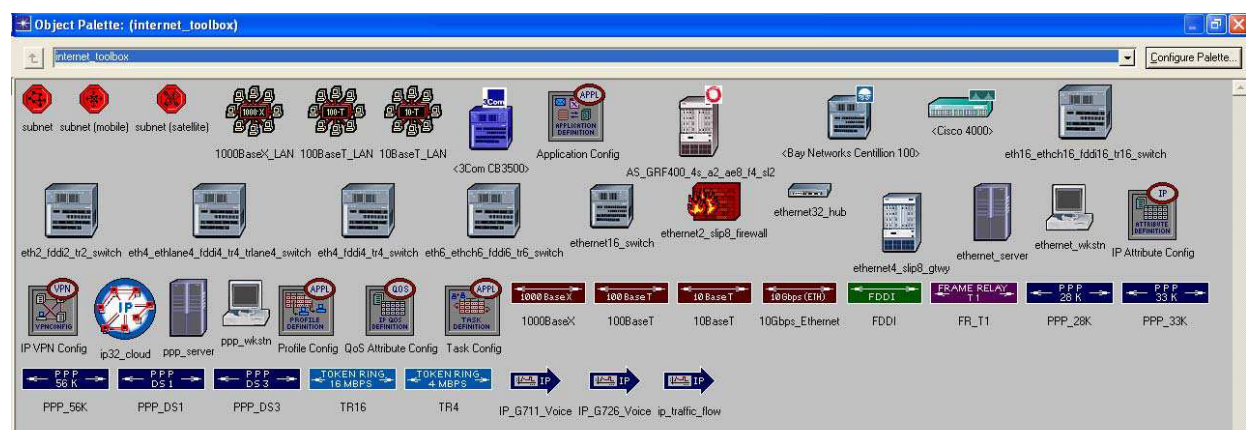

Figure 2.9: OPNET object palette.

This option makes your research for "components" more effective and it also allow you to select the exact server and/or router (3Com, Cisco, ACE...) that you are looking to use in your network.

- Configuring Applications: This step is used to define the profiles and applications that will be used by the LAN before you even begin constructing your network.

1. A Profile is applied to workstation, server, or LAN. It specifies the applications used by a particular group of users. You can have multiple profiles (heavy use of email or light use of file transfer) for multiple groups.

2. An Application may be any of the common applications (email, file transfer...) or could be an application that you can define yourself. OPNET comes standard with eight pre-defined applications: database access, email, file transfer, file print, Telnet session, video conferencing, Voice over IP call, and web browsing.

The next few steps are dedicated to creating that background utilization. The first model will act as a reference and the second one (with background utilization) will help to compare the results from the two scenarios. The network study shows that traffic rises Fig. 2.10 gradually over the course of the day as employees arrive and begin to work and then decrease rapidly as soon as the background utilization decreases. 


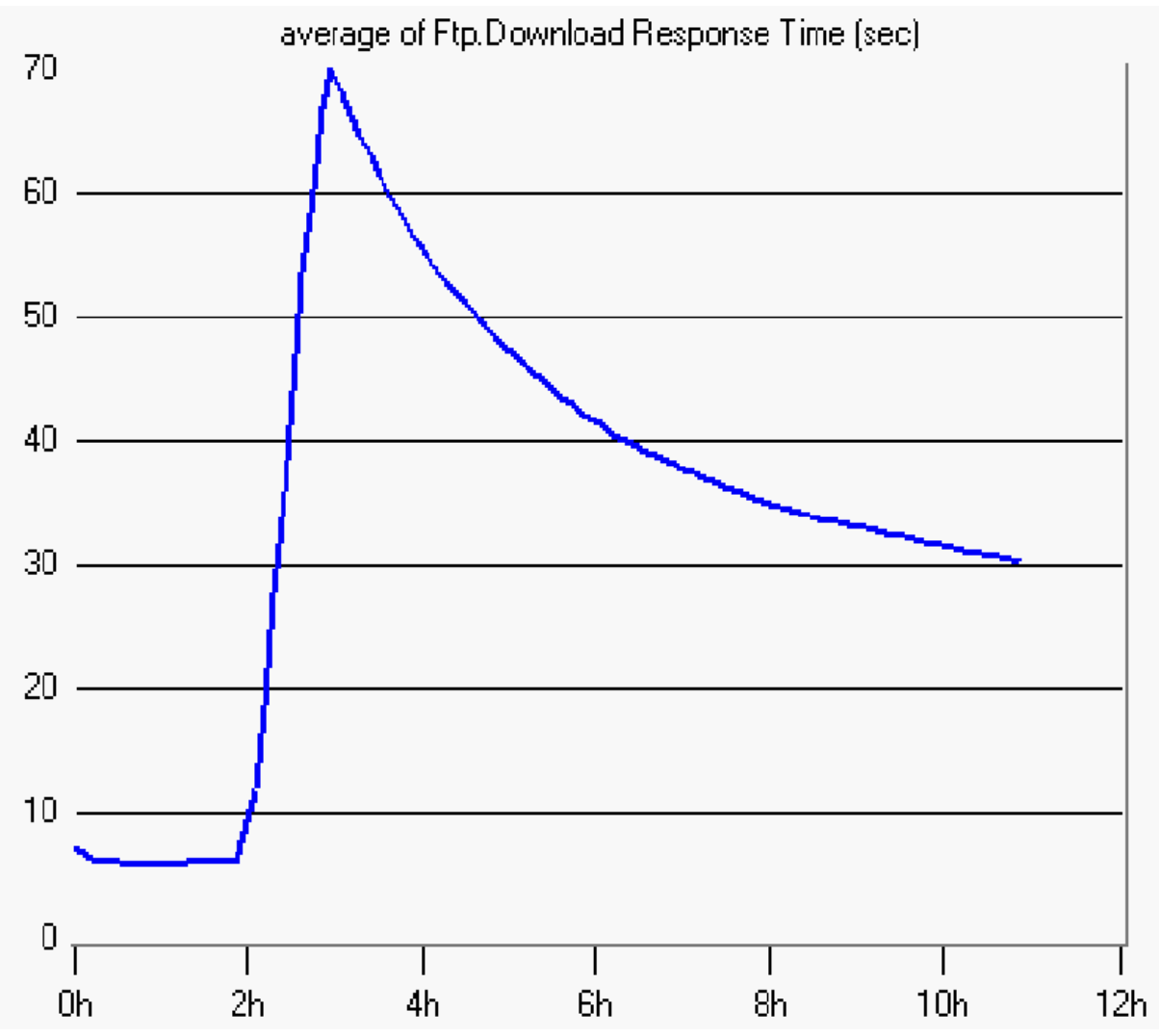

Figure 2.10: Traffic over the course of the day.

\subsection{Conclusion}

OPNET is a very useful tool for modeling of communication networks. Its built-in library and various pre-defined components allow one to define and study the network in an effective manner. Furthermore, its interface, speed and its very effective way to represent results make OPNET Modeler a "must have" tool when studying communication networks. 


\section{Chapter 3}

\section{The Two Area Four Generator System}

\subsection{Description}

Electro-mechanical oscillations between interconnected synchronous generators are problems inherent to power systems. The oscillations associated within a group of generators are called "inter-area modes" [15]. The inter-area modes of oscillation are affected by many factors such as the types of controllers, the loading conditions, etc... Therefore making them hard to study and control. An easy way to study inter-area modes of oscillations is to scale down the system from a large and complex power system to a smaller one that keeps the same characteristics. The complexity of the system model necessary to determine the stability of the system obscures the nature of the inter-area modes of oscillation. Therefore, in order to be able to study only those specific effects, one should construct a small but realistic system. A benchmark system has been suggested in the literature to study interarea oscillation. This system is a "two area systems". A special case of two area system is the two-area four generator system, also called " $2 \mathrm{~A} 4 \mathrm{G}$ ". The system configuration can be described as follows [16] [17]:

- Two sets of two generators on each side of the grid separated by a total distance of 220 $\mathrm{km}$. On each side, the generators are $20 \mathrm{~km}$ apart from each other. Each generator is connected to the grid via a tie line. Every single generator is equipped with the same simple exciter and all the generators have the same configuration. 
- A total of 2 loads are attached, on each side, to the system. The power can be flowing across the system in either direction. A total of 12 buses are used between the controller, the four generators, and the loads.

- Several control strategies have been proposed, including the use of a Static Var Compensator (SVC) controller, a Power System Stabilizer (PSS) controller, a Unified Power Flow Controller (UPFC), and Thyristor Controlled Series Capacitor (TCSC) controller. Placement of these controllers depends on different factors such as transmission capacity, control and stability objectives, etc...

\subsection{Why Use The 2A4G System?}

The $2 A 4 G$ (see Fig. 3.1) is a specially designed system that is used to study the ability of the overall system to withstand the loss of a line and/or unwanted variations in other operating conditions. The stability of the system has been tested and a control strategy has been implemented [16]. It appears that one PSS controller by itself or the combination of one PSS controller and local measurement are not enough to stabilize the system. This implies that the PSS controller might need remote measurements.

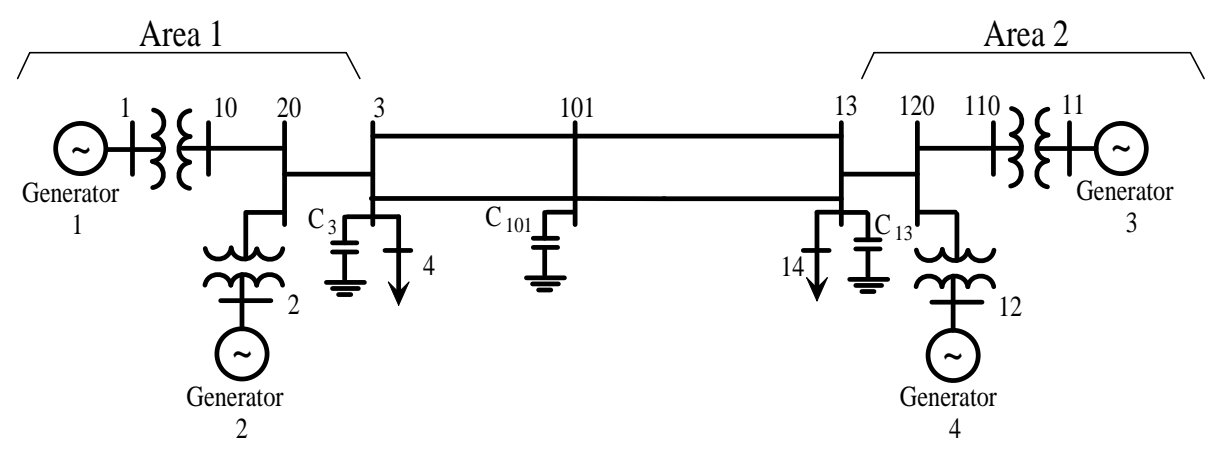

Figure 3.1: The Two Area System.

There are two different strategies that can be used to control the system. The first strategy is to place a SVC controller at the center of the grid and "feed" it with measurements from remote generators. The second strategy, the strategy that is going to 
be implemented later in the chapter, is to place a PSS controller at one generator and "feed" it with remote measurements. This strategy is implemented later in this chapter because it is the strategy that generates the longest delays. The main advantage of this method is that the controller is implemented based on the worst case scenario making it more likely to work in a better case scenario.

\subsection{Case Study with OPNET}

In the following case study, the PSS controller will be placed at the first generator within the grid and will receive remote measurements from all the other generators generating the longest delays. OPNET Modeler will be used to characterize the delays and number of packets dropped between each node [14] in order to determine the communication within the network. The results obtained using OPNET Modeler will later be used to characterize the effects of those delays on a real-time closed loop system. The complete case study for the $2 \mathrm{~A} 4 \mathrm{G}$ is described later in this chapter.

\subsubsection{Terminology}

Each area is composed of different elements that play a particular role in the transfer of data within the network [10]. The network is also capable of supporting various type of applications that generate traffic. All those important terms are defined below:

\section{Elements:}

- Router: A router is an intermediate device that connects communication links. A router takes information arriving on one of its incoming communication links and then forwards it on one of its outgoing communication link.

- Switch: A switch is a high performance multi-interface bridge. Like a bridge, a switch forward and filter frames using LAN destination addresses. A switch has a dozen interfaces that it can interact with.

- FTP: A FTP (File Transfer Protocol) is a protocol used to transfer file between a client and a server. FTP permits the user to issue two basic commands for transferring 
a file: "get" and "put". The "get" command triggers the transfer of a file from a remote server. The "put" command sends a file to a remote server. For connectionoriented transport protocols, (e.g. TCP), a new transport connection is opened for each file transfer. TCP is the default transport protocol for this application. The computation of the rate of file transfer is as follows:

$$
R_{t}=\frac{N}{L}
$$

where $R_{t}$ is the transfer rate, $\mathrm{N}$ is the number of file transferred, and $\mathrm{L}$ is the length of traffic captured.

- Connection: A connection is an interconnecting circuit between two or more locations for the purpose of transmitting and receiving data. A link can be either electrical or fiber optical cable (multiple different connection types and connection speed are available). For example, the PPP base is a point-to-point link that connects two nodes running IP (e.g., gateways) at a selectable data rate.

\section{Applications:}

- Remote Login: During a remote login application, or Telnet, the users login to different machines, interact with the operating systems of the remote hosts. The command they enter and the feedback they receive generate traffic on the network. The TCP protocol is the default protocol for the remote login application.

- Video Conferencing: A video conferencing application lets users transfer streaming video frames across the network. UDP is the default protocol used for video conferencing.

- Database: A database application enables the user to store information. Database operations are divided into two categories: a database entry, and a database query. A database entry results in a fixed amount of data being written into the database. A database query results in the client issuing a query, and the server responding with some data. The default transport protocol for the database application is TCP. 


\subsubsection{Modeling the Network:}

First, one needs to make sure that the distances between the areas are respected. Then, one needs to define what type of connections are going to be compared in the different scenarios. The different data rates that are going to be used to model the system are $56 \mathrm{kbps}$ (56K link), 1.544 Mbps (DS1), 44.736 Mbps (DS3), 100 Mbps (100BaseX), and 594.43 Mbps (OC-12) [18] [19]. Each scenario will serve as a benchmark for the next one. Comparing the results obtained will help one decide upon the optimal solution for the $2 \mathrm{~A} 4 \mathrm{G}$ system. The only two assumptions made in the case study are that the power used to transmit the signal is large enough to avoid the use of repeaters within the network. The other assumption is the losses over the transmission medium are negligible.

Each area will contain a switch, an FTP server, 25 workstations, connected to each other in a star configuration, connected with a 1000BaseX (1000 Mbps) connection, and a router (see Fig. 3.2).

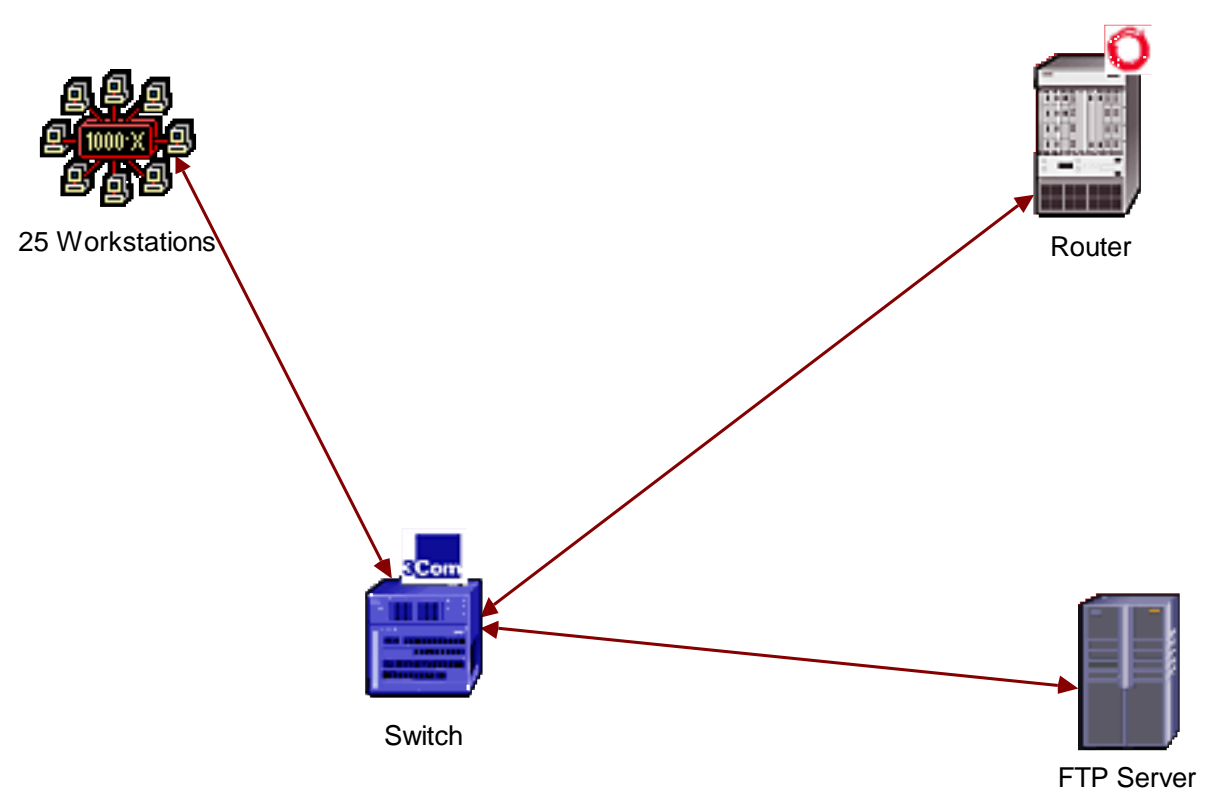

Figure 3.2: Detail of an Area.

The PSS controller will be characterized as a combination of a switch connected to a router and 6 servers (see Fig. 3.3). The servers are divided into 2 different sets that 
can perform the same tasks. The first set will be used as the main servers allowing data to be transferred from a remote area and back to the controller. The second set of servers will be used as a backup set.

All the routers will be connected together and then traffic will be added to the network. The transmitted packets will have the same format and they will use the same protocol to be transmitted but they will two different lengths (4500 bits and 400 bits). Having two different length allows to characterize the effect of the size of the packets on the transmission delays.

The next step is to setup the background utilization. The background utilization will be the factor that will strain the communication within the network, having a direct effect on the delays measured. By including background utilization, the case study becomes very close to a real system. The three different background utilizations compared are as follows: $10 \%, 50 \%$, and $90 \%$. The applications available to the engineers are the ones described in table 3.1 .

Table 3.1: Applications.

\begin{tabular}{|c|c|}
\hline Application & Use \\
\hline Email & heavy \\
File transfer & heavy \\
Database access & heavy \\
File print & light \\
Telnet session & light \\
Video conferencing & light \\
Voice over IP & light \\
Web browsing & heavy \\
\hline
\end{tabular}

Each application is supported by the network, and is available to every engineer working in the office. All applications will be executed simultaneously creating more stress on the network. The network is described in Fig. 3.4.

\subsection{Results}

The results we are seeking here are the average communication delays, the maximum communication delays, and the delay jitter for each communication link and for all of the three background utilizations (between the PSS controller and each remote area of 


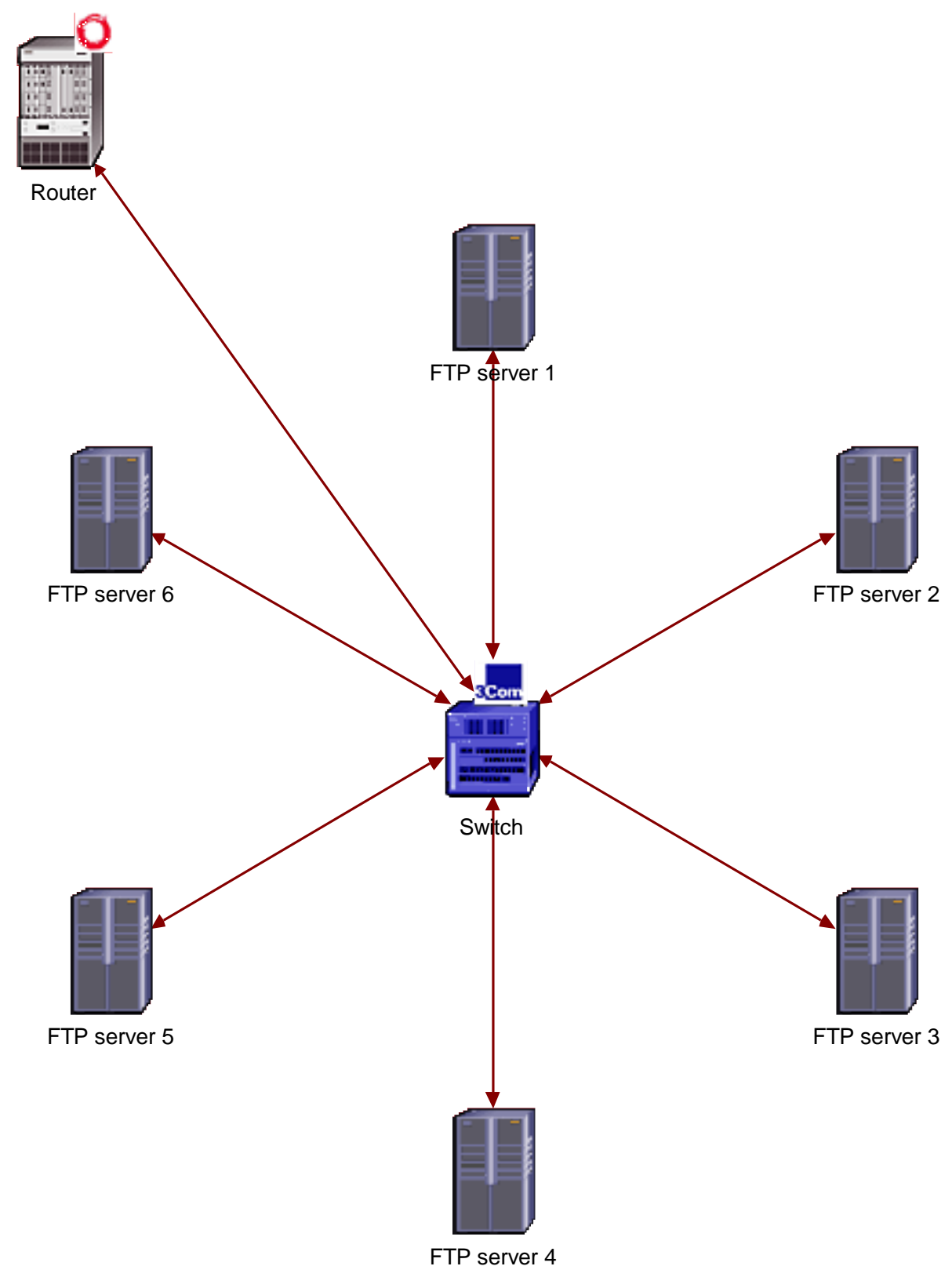

Figure 3.3: OPNET Model for the PSS Controller. 


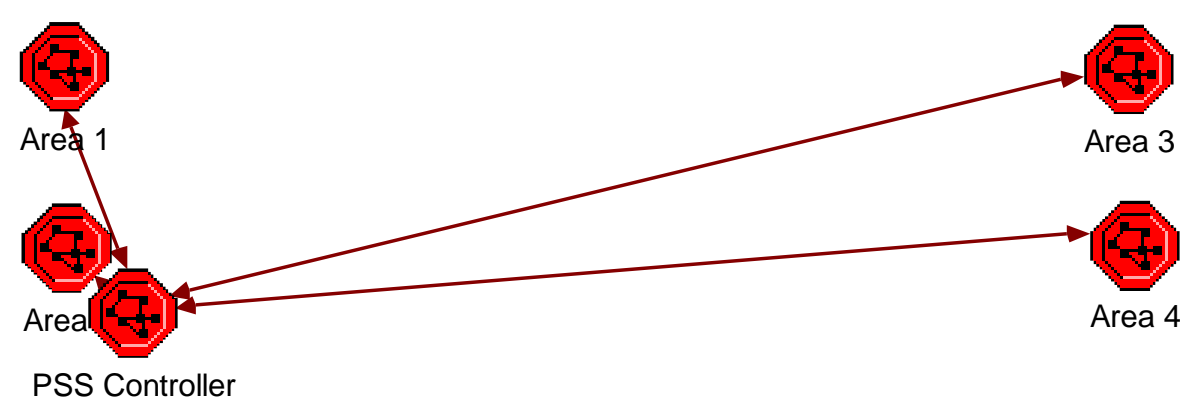

Figure 3.4: 2A4G OPNET network.

Table 3.2: Number of Packets dropped for each type of connection.

\begin{tabular}{|c|c|}
\hline Link & Packets Dropped \\
\hline Area 1 to PSS Controller & 0 \\
\hline Area 2 to PSS Controller & 0 \\
\hline Area 3 to PSS Controller & 0 \\
\hline Area 4 to PSS Controller & 0 \\
\hline
\end{tabular}

the network). We are also looking for the number of packets dropped during a day of work in the office. All those results are important to the project because they will be used later when implementing a second controller used to stabilize the system.

\subsubsection{Packets Dropped:}

The results obtained for each type of connection are summarized in table 3.3. It shows that, despite the background utilization, even the link that has the lowest performance (e.g. 56K line) still manages not to drop a single packet. This result is due to the fact that there is not enough strain on the network elements because of its relative small size. The traffic generated is not significant enough to force the routers to drop packets.

\subsubsection{Delays:}

The results obtain with OPNET Modeler are summarized in table 3.3 and are expressed in millisecond. The results obtained indicate that the delays are proportional to the capacity of the links and also to the background utilization. The length of the packet has no effect on the delay values. The small size of the network does not generate enough strain 
Table 3.3: Delays between remote generators and the PSS controller.

\begin{tabular}{|c|c|c|c|c|c|c|c|c|c|c|c|}
\hline & \multicolumn{2}{|c|}{ Utilization } & \multicolumn{3}{|c|}{$10 \%$} & \multicolumn{3}{c|}{$50 \%$} & \multicolumn{3}{c|}{$90 \%$} \\
\hline Link & \multicolumn{2}{|c|}{ Delay $(\mathrm{msec})$} & Avg. & Jitter & Max & Avg. & Jitter & Max & Avg. & Jitter & Max \\
\hline \hline $56 \mathrm{~K}$ & Packet & 400 & 117.2 & $5.8 \%$ & 124.1 & 117.5 & $7.9 \%$ & 125.8 & 129.1 & $8.8 \%$ & 158.1 \\
& Size & 4500 & 118.0 & $7.9 \%$ & 127.8 & 118.3 & $7.9 \%$ & 129.9 & 131.9 & $9.1 \%$ & 152.2 \\
\hline \multirow{2}{*}{ DS-1 } & Packet & 400 & 83.7 & $4.9 \%$ & 88.7 & 84.5 & $5.1 \%$ & 88.9 & 92.0 & $5.2 \%$ & 97.1 \\
& Size & 4500 & 84.0 & $5.0 \%$ & 89.2 & 84.4 & $5.0 \%$ & 89.6 & 92.0 & $5.3 \%$ & 97.5 \\
\hline DS-3 & Packet & 400 & 31.1 & $4.6 \%$ & 33.9 & 31.5 & $4.7 \%$ & 32.8 & 33.9 & $5.0 \%$ & 37.5 \\
& Size & 4500 & 30.9 & $4.8 \%$ & 34.2 & 31.6 & $4.7 \%$ & 33.0 & 34.2 & $5.0 \%$ & 37.8 \\
\hline 100 & Packet & 400 & 13.2 & $3.9 \%$ & 13.9 & 13.3 & $4.2 \%$ & 13.6 & 15.3 & $6.9 \%$ & 18.1 \\
BaseX & Size & 4500 & 13.1 & $4.1 \%$ & 13.9 & 13.3 & $4.1 \%$ & 13.8 & 15.9 & $7.2 \%$ & 18.5 \\
\hline OC-12 & Packet & 400 & 1.3 & $2.0 \%$ & 5.9 & 1.3 & $2.1 \%$ & 5.6 & 1.3 & $3.3 \%$ & 5.8 \\
& Size & 4500 & 1.2 & $1.9 \%$ & 5.5 & 1.3 & $2.1 \%$ & 5.7 & 1.4 & $3.2 \%$ & 6.1 \\
\hline
\end{tabular}

on the router within the network to make the size of the packets a factor that influences the value of the delays.

The delays are minimum for a fiber optic cable used with $10 \%$ of background utilization (average delay of $1.25 \mathrm{msec}$ and maximum delay of $5.9 \mathrm{msec}$ ). The delays are maximum for the PPP 56K link used with $90 \%$ of background utilization (average delay of $130.5 \mathrm{msec}$ and maximum delay of $158.1 \mathrm{msec}$ ). The results obtained are the ones expected. It is important to emphasize the consistency of the results proving their quality.

A crucial component of end-to-end delay is the random queuing delays in the routers. Because of these varying delays within the network, the time from when a packet is generated at the source until it is received at the receiver can fluctuate from packet to packet. This phenomenon is called jitter. Jitter is the variance in one-way latency and is calculated based on sending and receiving time stamps of consecutive packets sent out.

Let $\mathrm{N}$ be the number of transmitted packets and $\tau_{i}$ be the delay of the $i^{t} h$ packet, $1 \leq i \leq N$. The average delay, $\bar{\tau}$ is given by:

$$
\bar{\tau}=E[\tau]=\frac{1}{N} \sum_{i=1}^{N} \tau_{i}
$$


and the equation of the delay jitter $\sigma^{2}$ is given by:

$$
\sigma^{2}=E\left[(\tau-\bar{\tau})^{2}\right]=\frac{1}{N-1} \sum_{i=1}^{N}\left(\tau_{i}-\bar{\tau}\right)^{2}
$$

In order to be able to obtain the delay jitter in percent, one needs to normalize with respect to $\bar{\tau}$. In OPNET Modeler, the delay jitter is calculated using time stamps of every two consecutive packets.

Delay and delay jitter are the important measures of Quality of Service (QoS) particularly for the traffic in the network environment with bursty background traffic. The network conditions including traffic load, traffic burstiness and burst-length all have significant effects on delay and delay jitters.

\subsection{Real-time closed loop system}

In this section, we will see the implementation of a controller for a real-time closed loop system [17]. This implementation will be based upon the results obtained in the previous section. The simulation will be executed with Simulink.

\subsubsection{System Representation:}

The system representation in the Simulink workspace is shown in Fig. 3.5. The system is first expressed with the state space equation [17].

$$
\begin{aligned}
& \dot{x}(t)=A x(t)+B u(t) \\
& y(t)=C x(t)+D u(t)
\end{aligned}
$$

where $\mathrm{A}$ is a matrix of size $40 \times 40, \mathrm{~B}$ is $40 \times 1$, C is $1 \times 40$, and $\mathrm{D}$ is 0 . A, B, C, and D contain the generators and exciters dynamics of the whole power system. If one would have decided to use a SVC controller (located in the center of the grid) instead of a PSS controller, D would not be composed of zeros. The linear transfer function $\mathrm{H}(\mathrm{S})$ of the continuous system $2 \mathrm{~A} 4 \mathrm{G}$ is obtain by using the SS2TF function into Matlab [17]. The SS2TF function allows 
Table 3.4: Stability of the System.

\begin{tabular}{|c|c|}
\hline Delay & System \\
\hline Delay $\leq D_{c}$ & Stable \\
Delay $>D_{c}$ & Unstable \\
\hline
\end{tabular}

one to convert state-space filter parameters to transfer function form.

$$
\begin{aligned}
H(S) & =C(s I-A)^{-1} B \\
& =\frac{|(s I-A+B C)|-|(s I-A)|}{|(s I-A)|}
\end{aligned}
$$

where $|(s I-A)|$ is the determinant of the matrix (sI-A).

\subsubsection{Results:}

The system is simulated for a time duration of 15 seconds using Simulink. As a benchmark we will use the system that does not have any delays. The rest of the simulations use the delays obtained with OPNET Modeler. The results are presented in figure 3.6. The output obtained is the speed of the shaft, " $\omega_{2}$ ", of the second generator as a function of time. It is obvious that for the delays obtained the system is still controllable. The system becomes uncontrollable when the delays reach the critical delay value $D_{c}$ of $0.437 \mathrm{sec}$ (see fig. 3.7). The value of $D_{c}$ is almost 2.77 times bigger than the maximum delay value obtained. The results are summarized in table 3.4. Even if the $56 K$ link is "fast" enough, it should be a link dedicated to the controller's information only. The margin between the maximum delay of the worst scenario and the critical delay is big enough to assume that even if the network expends, the controller will be able to stabilize the system.

\subsection{Conclusion}

The Two Area Four Generator system is a benchmark system that can be used to study the inter-area modes of oscillations. OPNET Modeler as a tool can help modeling the delays generated within the network and therefore improve the design of the controller for such a system. 
When designing a controller for the $2 \mathrm{~A} 4 \mathrm{G}$ system one has to make sure that the links selected to carry the information from one area to another are fast enough to generate delays smaller than the critical delay value.

If the network comes to expend, one should expect an increase in the maximum delay. In order to compensate this problem, one should evaluate the need for the use of a "fast" link in the design of the expansion of the network of controlled element. 


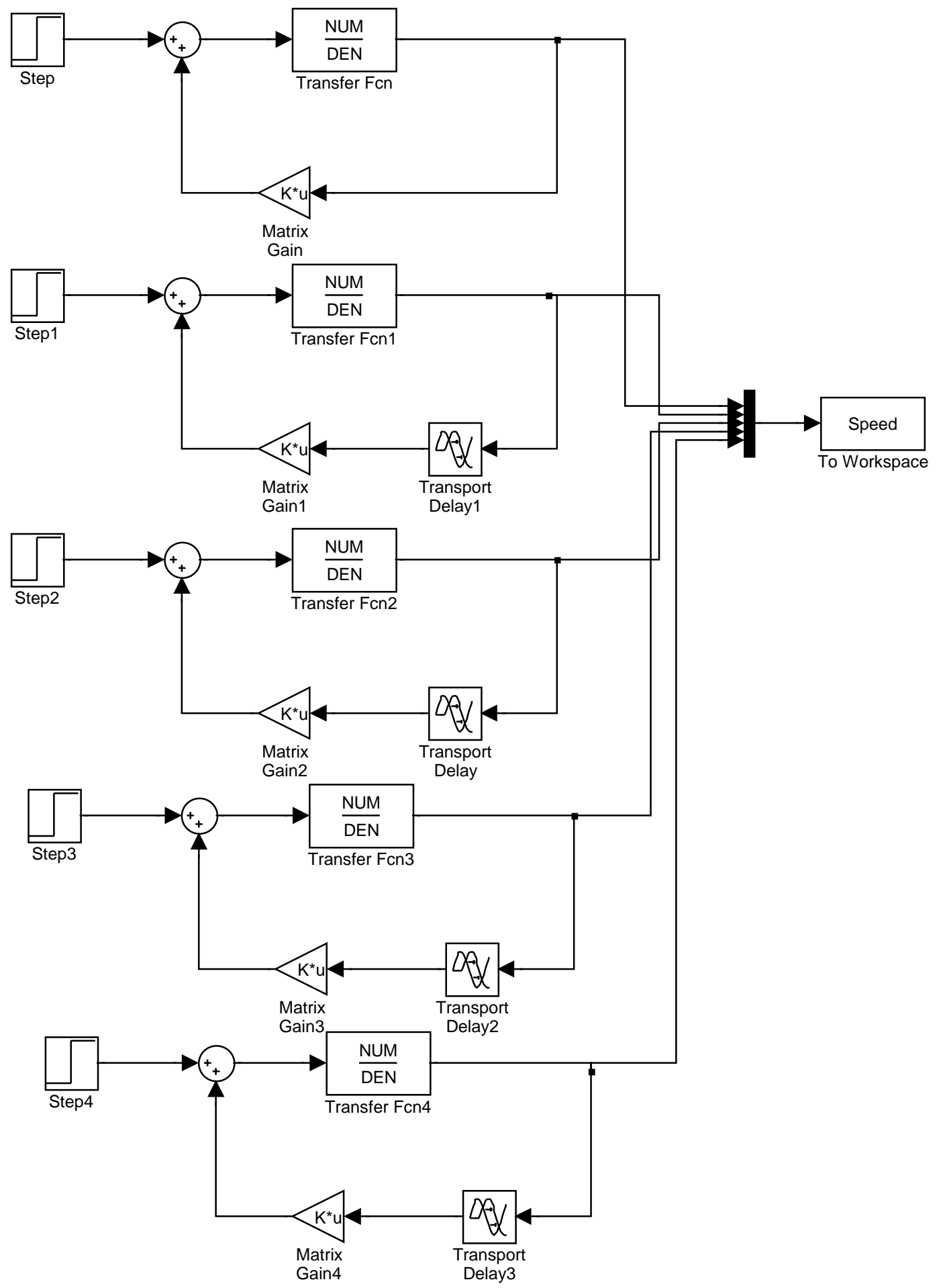

Figure 3.5: Representation of the system with Simulink. 


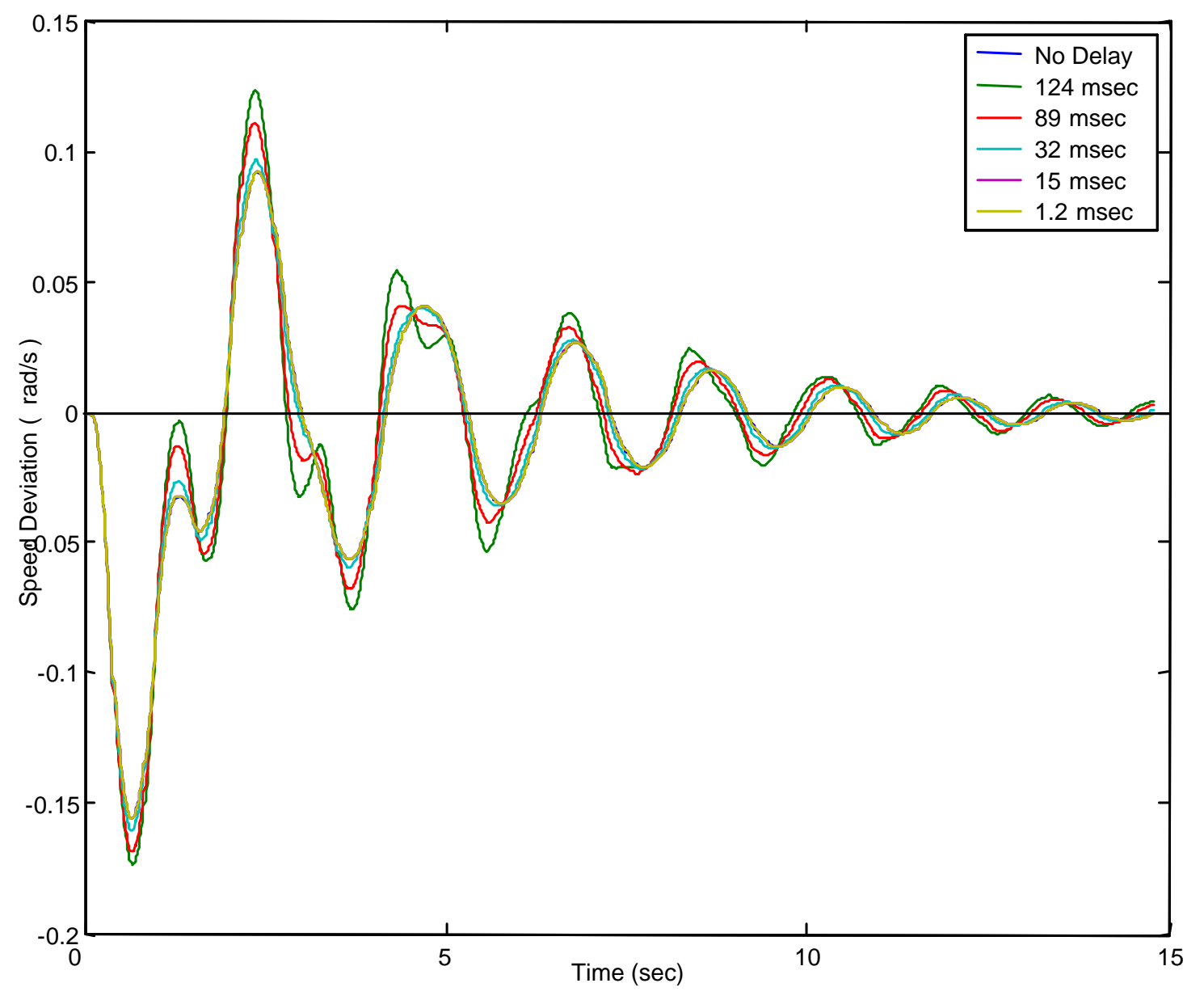

Figure 3.6: Speed of the Shaft w2 as a function of the delays. 


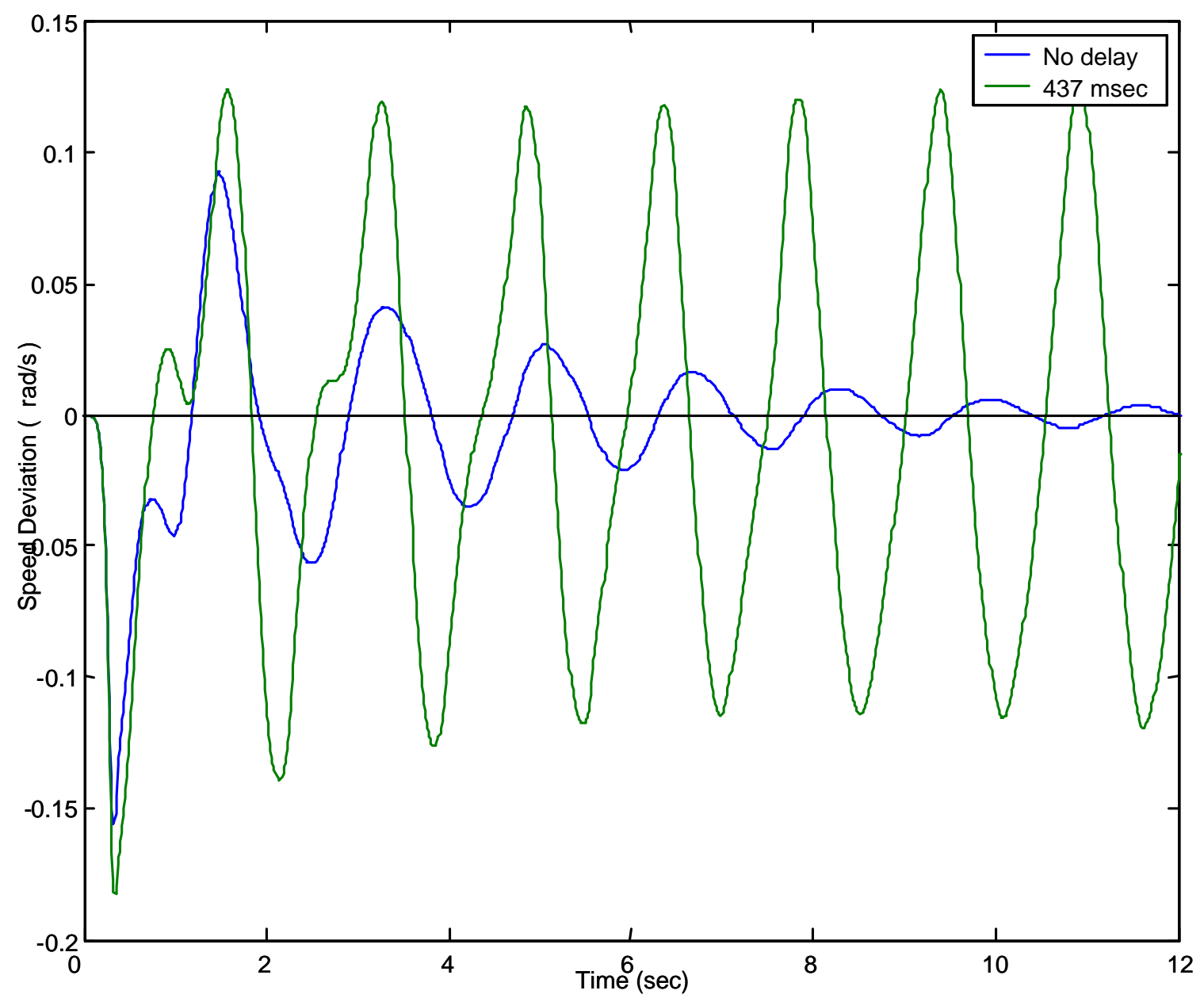

Figure 3.7: Speed of the Shaft w2 as a function of the delays (when delay bigger than Dc). 


\section{Chapter 4}

\section{Other Solutions}

\subsection{Dedicated Fiber Optic Network}

\subsubsection{Introduction:}

Fiber systems are now very common, and new installations and applications appear frequently. Fiber optic communications were developed quickly after the first low-loss fibers were produced in 1970. The growth has been maintaining for many years. Around 1984, fibers were used mainly to deliver telephone messages between major cities. In 1988, the land-based long-distance fiber telephone network was nearly complete. The submarine fiber telephone cables were being installed beneath the oceans. In addition, researchers were working on fiber optic LANs. A few years after this, the installation of fiber LANs was increasing at a rapid rate. Also tremendous efforts were realized for bringing fiber to all homes, holding the promise for extended services to the individual subscriber.

\subsubsection{Definitions:}

- Fiber Optic Cable: The basic material for glass fibers is silicon dioxide. A fiber optic cable is a fairly flexible cable, light, and easy to install. Compared to copper cables, fiber optics have many advantages. A few of them are the immunity to electromagnetic interferences and a very low fiber-to-fiber cross talk. Optic fibers are insulators meaning that no current flows through them [18]. 
Table 4.1: Power attenuation in $\mathrm{dB} / \mathrm{km}$.

\begin{tabular}{|c|c|}
\hline Frequency $(\mathrm{Hz})$ & Attenuation $(\mathrm{db} / \mathrm{km})$ \\
\hline $3.6 \mathrm{e} 14$ & 2.5 \\
$2.45 \mathrm{e} 14$ & 0.5 \\
$1.8 \mathrm{e} 14$ & 0.2 \\
\hline
\end{tabular}

- Dedicated Fiber Optic Network: A dedicated fiber optic network is a high-speed, dedicated point-to-point connectivity for voice, data and video applications. Typically consisting of non-switched communications circuits and the required equipment to connect two or more locations. The main features of this dedicated network are high-capacity bandwidth from DS-1 to OC-X, 100\% network availability, customized circuits between locations [20].

\subsubsection{Signal Degradation and Delay:}

Light rays cannot travel over a very long distance in a fiber optic cable. In order to maintain a level of BER sufficient, repeaters need to be introduced periodically on the network. Typical fiber losses are around $4 \mathrm{~dB} / \mathrm{km}$ [19] when operating at wavelength around $0.82 \mathrm{~m}$. Nowadays, fiber losses are down to only few tenths of a dB/km, and available for use around $1.4 \mu \mathrm{m}$. Signal degradation in fiber optic communication can take place due to the following reasons.

- Attenuation: Absorption, scattering and radiative losses of the optical energy are the main reasons for attenuation of light signal (see table 4.1).

The coefficient of attenuation (in $\mathrm{dB} / \mathrm{km}$ ) [21] is expressed as follows:

$$
\alpha=\frac{10 \log \frac{P_{\text {in }}}{P_{\text {out }}}}{L_{f}}
$$

where Pin is the input optical power, Pout the output optical power and L the length of the fiber.

- Fiber Materials: The small difference of refractive index (around 1\%) between the core and the cladding allows avoiding losses. In order to do so, the two materials need 
to have almost the same amount of doping material (fluorine and various oxides). The dopants of the fiber can lead to scattering of light, thus loss of signal power [22].

- Absorption: Absorption is mainly due to the fiber material. It is caused by the atomic defects in the composition of the glass. Those defects can be characterized in two different types: intrinsic absorption by the basic constituents of the fiber, extrinsic absorption by impurity atoms in the glass.

- Delay: In fiber optic communication most of the delays occur at the repeaters. The passage of the light through the medium (the fiber) is almost instantaneous (at the speed of light, around $3.54 \mathrm{e} 8 \mathrm{~m} / \mathrm{s}$ ) but the passage of the information through the repeaters can take up to few tenths of milliseconds [23].

\subsubsection{Technical Data:}

- Signal Processor: In the case of analog transmission, signal processor consists of amplifying and filtering of the signal. All the undesired frequencies should be blocked in order to obtain better clarity in the transmitted signal. All the frequencies not carrying any information should be prevented from further travel along the fiber. Reaching a high quality in filtering allows one to maximize the signal-to-noise ratio (SNR). Moreover, signal processor could include a decision making circuit. The circuit helps deciding if the received bit is a 0 or a 1 . Because of the presence of noise, and in order to be able to have a good quality transmission, a very low bit-error-ratio (BER) needs to be achieved.

- Bandwidth: One of the most important characteristics when transmitting a signal is the allocated bandwidth. The table 4.2 summarizes the bandwidth requirement of several analog systems. Fiber optics can easily operate at rates higher than a couple of $\mathrm{GHz}[21]$. In order to transmit an analog signal one needs to respect the sampling theorem. The theorem states that for an analog signal that is transmitted digitally, the bit rate depends on the rate at which the signal has been sample. The sampling 
Table 4.2: Data rates for different fiber optical cables.

\begin{tabular}{|c|c|}
\hline Designation & Data Rate (Mbps) \\
\hline OC-1 & 51.84 \\
OC-3 & 155.52 \\
OC-12 & 622.08 \\
OC-24 & 1244.16 \\
OC-48 & 2488.32 \\
\hline
\end{tabular}

rate needs to be a least twice as big as the highest frequency that contain the signal. In order to improve the amount of information transmitted within one signal, one needs to combine information within the same information channel. This operation is called multiplexing messages, and could be described as the process of interleaving the data bits at the transmitter.

\subsubsection{Conclusion:}

Optic fibers are extremely easy to use and very reliable. Their significant bandwidth capacity allows high data rate. This additional bandwidth could be easily sold to a utility company in order to provide additional services. A dedicated fiber optic network is a good solution to the $2 \mathrm{~A} 4 \mathrm{G}$ problem allowing high data rate and good reliability to the users.

\subsection{Virtual Private Network}

A Virtual Private Network (VPN) is a secure private network solution that lets growing and large businesses connect offices, partners, suppliers and remote employees without a heavy investment in infrastructure or personnel. VPN uses the speed and capacity of the Internet to provide secure connections at a lower cost than other WAN technologies. A VPN offers a innovative approach to the traditional problem of supplying efficient, reliable, user-friendly telecommunication tools. Multiple options of configuration and access speeds (from X-DSL to T-3) are available (you can even add remote access capabilities).

The main advantage of a VPN is to lease private trunks of a public or private 
network to interconnect all the remote locations [24]. A VPN is like a "pipe within the pipe" (see Fig. 4.1).

Conceptually, a VPN retains most of the advantages of the private network without the

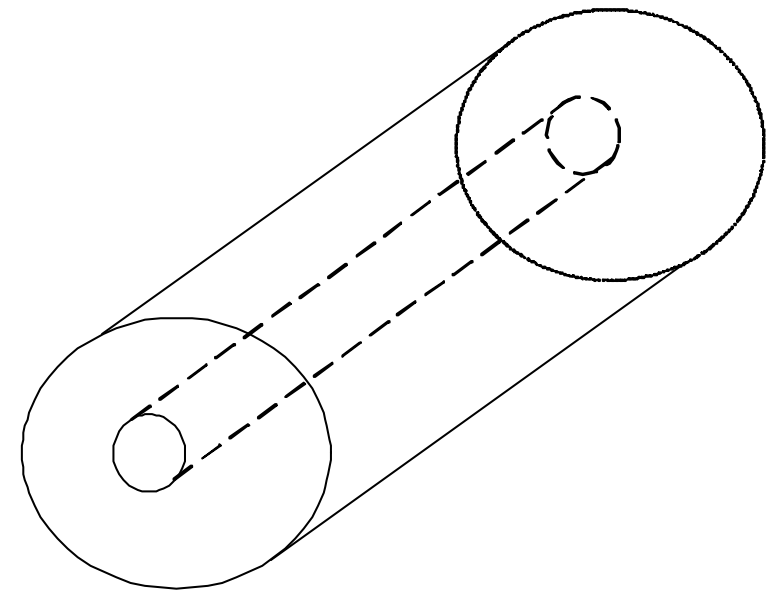

Figure 4.1: A Virtual Private Netwok: A "pipe within the pipe".

operation difficulties. The VPN uses, in a time sharing manner with other traffic, the resources of a public or private network. The maintenance and operating aspects are left to the charge of the network operator. Additional benefits of the VPN are the increase of the reliability, flexibility, and performance. The users are free from some of the ties to existing dedicated technology, and all the new features are available to the user immediately.

\subsection{File Transfer Protocol}

\subsubsection{Introduction:}

The IP is the method used to send data from one computer to another on the Internet. Each computer on the Internet has one IP address that uniquely identifies it from all other computers on the Internet. When you send or receive data, the message gets divided into smaller chunks called packets. Each of these packets contains both the sender's Internet address and the receiver's address. A packet is first sent to a gateway computer that only understands a small part of the Internet. The gateway computer reads the destination address and forwards the packet to an adjacent gateway that reads the destination address and so forth across the Internet until one gateway recognizes the packet as belonging to a computer within its immediate neighborhood or domain. The gateway at the end of the 
chain forwards the packet directly to the computer whose address is specified. Because a message is divided into a number of packets, each packet can, if necessary, be sent by a different route across the Internet. Packets can arrive in a different order than the order they were sent in. The Internet Protocol just delivers them. It is up to the TCP protocol to put them back in the right order [10].

IP is a connectionless protocol. There is no continuing connection between the end points that are communicating. Each packet that travels through the Internet is treated as an independent unit of data independent to any other unit of data. The TCP is the only reason why the packets do get put in the right order. The TCP is the connection-oriented protocol. The TPC protocol keeps track of the packet sequence in a message. In the OSI communication model, the third layer, the networking layer, is the IP layer.

\subsubsection{IPv6:}

The most widely used version of IP today is Internet Protocol version 4 (IPv4). However, IP version 6 (IPv6 is the latest level of the IP) is also beginning to be supported. The most obvious improvement in IPv6 over the IPv4 is that IP addresses are lengthened from 32 bits to 128 bits. This extension anticipates considerable future growth of the Internet and provides relief for what was perceived as an impending shortage of network addresses.

IPv6 has also been called "IP Next Generation" (IPng). IPv6 was designed as an evolutionary set of improvements to the current IPv4. Network hosts and intermediate nodes with either IPv4 or IPv6 can handle packets formatted for either level of the Internet Protocol [24]. Users and service providers can update to IPv6 independently without having to coordinate with each other.

IPv6 has rules for three different types of addressing: unicast (one host to one other host), anycast (one host to the nearest of multiple hosts), and multicast (one host to multiple hosts). The main advantages [24] of IPv6 are:

1. Options are contained in an extension of the header that is examined only at the 
destination, thus speeding up overall network performance.

2. The introduction of an "anycast" address gives the option of sending a message to the nearest of several possible gateway hosts with the idea that any one of them can manage the forwarding of the packet to others. Anycast messages can also be used to update routing tables along the line.

3. Packets can be identified as belonging to a particular flow so those packets can get assigned a higher priority allowing them to arrive in real time. This process provides a higher QoS relative to other customers.

4. The IPv6 header includes extensions that allow a packet to specify a mechanism for authenticating its origin, for ensuring data integrity, and for ensuring privacy and security.

\subsubsection{Conclusion:}

IPv6 gives you the ability to assign different QoS and priority classes. Thus, you could assign highest priority to the control information, be less susceptible to background applications, and ensure a higher privacy .

\subsection{Conclusion}

This chapter introduced the concept of dedicated fiber optic network as a solution to the $2 \mathrm{~A} 4 \mathrm{G}$ problem. Using the intrinsic qualities of a fiber optic cable one could easily design a network with short delays. The chapter then introduced the notion of virtual private network. Virtual private network can be used to provide secure, fast connections to remote areas that need to be inter-connected. The end of the chapter is dedicated to a new version of the IP protocol (IPv6). The main characteristics and advantages of the use of IPv6 are also given. 


\section{Appendix A}

\section{List of Variables}

$R_{t}$ Transfer rate

$N$ Number of file transferred

$L$ Length of traffic captured

$\bar{\tau}$ Average Delay

$\tau$ Delay

$\sigma^{2}$ Delay Jitter

$\alpha$ Coefficient of attenuation

$P_{\text {in }}$ Optical input power

$P_{\text {out }}$ Optical output power

$L_{f}$ Length of the fiber 


\section{Appendix B}

\section{Acronyms}

WCRL Wireless Communication Research Laboratory

APERC Advanced Power Engineering Research Laboratory

ISO Independent System Operator

RTO Regional Transmission Owner

$P X$ Power Exchange

$S C$ Schedule Coordinator

PSS Power System Stabilizer

$2 A 4 G$ Two Area Four Generator System

ARPA Advance Research Projects Agency

$W A N$ Wide-Area Network

$L A N$ Local-Area Network

ATM Asynchronous Transfer Mode

$B E R$ Bit Error Rate

OSI Open System Interconnections

ISO International Standards Organization 
ITU International Telecommunications Union

$I A B$ Internet Activities Board

TCP/IP Transport Connection Protocol/Internet Protocol

FTP File Transfer Protocol

VoIP Voice over IP

$M A C$ Media Access Control

$S V C$ Static Var Compensator

UPFC Unified Power Flow Controller

TCSC Thyristor Controlled Series Capacitor

QoS Quality of Service

$B E R$ Bit Error Ratio

NS2 Network Simulator version 2

$I E E E$ Institute for Electrical and Electronic Engineers

$V P N$ Virtual Private Network

SS2TF State Space Equation to Transfer Function

$S N R$ Signal to Noise Ratio

IPv4 Internet Protocol version 4

IPv6 Internet Protocol version 6

IPng Internet Protocol Next Generation 


\section{References}

[1] S. Kanhouwa, "Performance issues for a changing electric power industry." web peb, March 2003. http://www.eia.doe.gov/cneaf/electricity_pub_summaries/e_chg_sm.html.

[2] N. Biju, "Telecommunications for a deregulated power industry," Master's thesis, West Virginia University, 2002.

[3] W. Liggett, "The changing stucture of the electric power industry 2000: An update." web page, March 2003. http://www.eia.doe.gov/cneaf/electricity/chg_stru_update/update2000.html.

[4] P. customer service, "Pjm: The power of connecting." http://www.pjm.com, January 2003.

[5] W. Liggett, "Executive summary: Energy information administration electricity reform." web page, March 2003. http://www.eia.doe.gov/cneaf/electricity/ex_summ/summary.html.

[6] C. ISO, "Mission and goal." http://www.caiso.com/aboutus/mission.html, March 2003.

[7] C. ISO, "About APX: Who we are, what we do." http://www.apx.com/sHome_html/About_APX.html, March 2003.

[8] A. B. Carlson, P. B. Crilly, and J. C. Rutledge, Communication Systems: An Introduction to Signals and Noise in Electrical Communication. McGraw Hill, 4th ed., 2002.

[9] W. Stallings, Data and Computer Communications. Prentice Hall, 4th ed., 1996.

[10] J. F. Kurose and K. W. Ross, Computer Networking: A Top-Down Approach Featuring the Internet. Asia, Pearson Education, 2001.

[11] R. S. Raji, "Smart networks for control," IEEE Spectrum, vol. 18, pp. 49-55, June 1994.

[12] D. Wangerin, C. DeCoro, L. M. Campos, H. Coyote, and I. D. Scherson, "A molecular client-server discrete event simulator for networked computers," in Symposium on performance evaluation of computer and telecommunication systems, May 2001. 
[13] "Simulation software links: Discrete event simulation," 2002. http://www.topology.org/soft/sim.html.

[14] OPNET, "Introduction to OPNET modeler: Accelerating network R\&D," 2003. http://www.opnet.com/TechWorkshops/.

[15] M. Klein, G. Rogers, and P. Kundur, "A fundamental study of inter-area oscillations in power systems," in IEEE Transactions on Power Systems, vol. 6, pp. 124-136, August 1991.

[16] A. Hasanovic and A. Feliachi, "Genetic algorithm based inter-area oscillation damping controller design using Matlab," in Power Engineering Society, pp. 126-148, July 2002.

[17] A. Hasanovic, "A simulation and analysis toolbox for power systems and genetic algorithm based damping controller design," Master's thesis, West Virginia University, 2002.

[18] J. C. Palais, Fiber Optic Communications Fourth Edition. Prentice Hall, 4th ed., 1998.

[19] J. M. Senior, Optical Fiber Communications Principles and Practice. Prentice-Hall, 1984.

[20] X. web site, "Xo: Products and Services." http://www.xo.com/products, April 2003.

[21] G. Keiser, Optical Fiber Communications. McGraw-Hill, 1984.

[22] C. C. Wang and W. K. Burns, "Fiber-optic delay lines for broadband signal processing," Electronic Components Conference, vol. 39, pp. 365-371, May 1989.

[23] S. D. Personick, Optical Fiber Transmission Systems. New York and London: Plenum Press, 1981.

[24] D.Wood, V.Stoss, L.Chan-Lizardo, G.S.Papacostas, and M.E.Stinson, "Virtual Private Networks," Private Swtiching Systems and Networks, pp. 132-136, June 1998. 